\title{
Report of a Subcommittee on the Nomenclature of $n$-Dimensional Crystallography. I. Symbols for point-group transformations, families, systems and geometric crystal classes $\dagger$
}

\author{
T. Janssen, ${ }^{a}{ }^{*} \neq$ J. L. Birman, ${ }^{b}$ V. A. Koptsik, ${ }^{c}$ M. Senechal ${ }^{d}$ D. Weigel,${ }^{e}$ A. Yamamoto, ${ }^{f}$ S. C. Abrahams ${ }^{g}$ \\ AND TH. HAHN ${ }^{h}$ \\ ${ }^{a}$ Institute of Theoretical Physics, University of Nijmegen, NL-6525ED Nijmegen, The Netherlands, ${ }^{b}$ Department of \\ Physics, City University of New York, New York, NY 10031, USA, ${ }^{c}$ Institute of Physics, Moscow State University, \\ Moscow 119899, Russia, ${ }^{d}$ Department of Mathematics, Smith College, Northampton, MA 01063, USA, ${ }^{e}$ Laboratoire \\ de Chimie-Physique du Solide, Ecole Centrale Paris, Chatenay Malabry, F-92295 France, ${ }^{f}$ National Institute for \\ Research in Inorganic Materials, Namiki, Tsukuba, Ibaraki, Japan, ${ }^{g}$ Physics Department, Southern Oregon \\ University, Ashland, OR 97520, USA, and ${ }^{h}$ Institut für Kristallographie, RWTH Aachen, Aachen, D-52066 Germany. \\ E-mail: ted@sci.kun.nl
}

(Received 24 November 1998; accepted 25 November 1998)

\begin{abstract}
The notation of crystallography in arbitrary dimensions is considered. Recommended symbols for point-group transformations, geometric crystal classes, families and systems are presented.
\end{abstract}

\section{Introduction}

The derivation of all 230 three-dimensional space groups by Fedorov (1891a,b) and Schoenflies (1891) became the geometrical and group-theoretical basis for crystal structure analysis developed by both Braggs following the discovery of X-ray diffraction by von Laue in 1912. Space-group information based on these derivations became widely available to users in International Tables for Crystallography (1995) and its predecessor publications, hereafter ITC.

The mathematical formulation of the theory of space groups, for example by Bieberbach (1911), led naturally to their generalization in arbitrary dimensions. An algebraic treatment of the theory was given by Ascher \& Janner (1965, 1968). Lists of transformations, point groups, lattices and space groups were derived for specific higher dimensions. Special cases were treated by Heesch (1929), Hurley (1951, 1966) and Janssen (1969) for four-dimensional space. The first complete list of all four-dimensional space groups was established by Brown et al. (1978). Partial results in five, six and seven

$\dagger$ Established 23 February 1990 by the IUCr Commission on Crystallographic Nomenclature, with all members including A. J. C. Wilson (Chairman) and W. Opechowski appointed 7 May 1990, under general ground rules outlined in Acta Cryst. (1979), A35, 1072. A. J. C. Wilson resigned 1 July 1993, W. Opechowski 25 February 1992 and H. Wondratschek 10 August 1996. T. Janssen was appointed Chairman 1 July 1993. Professor Opechowski died 27 September 1993 and Professor Wilson 1 July 1995 . The Report was received by the Commission on 16 March 1998 and accepted 16 November 1998.

\$ Chairman. dimensions have been derived by Plesken \& Pohst (1977), Janner et al. (1983) and Plesken \& Hanrath (1984).

The increasing use of three-dimensional space groups for structure determination during the early decades of this century made a nomenclature that would be widely accepted necessary. The notations of Schoenflies and of Hermann-Mauguin successfully filled this need. The former is often used by spectroscopists, the latter by crystallographers. Such a notation for higher dimensions is presently missing, although the number of fields in which the use of specific space groups in higher dimensions has become essential has increased steadily. This situation holds not only for mathematics where, for example, certain groups are associated with spaces of constant curvature but increasingly in describing the symmetry of aperiodic systems such as quasicrystals (Yamamoto, 1996). Although a complete overview of all possible applications is not yet available, it is useful to consider a unified nomenclature before the diversity of the fields and usages leads to widely different de facto nomenclatures and notations for the same objects. Concern for this situation led the Commission on Crystallographic Nomenclature of the International Union for Crystallography to establish a Subcommittee to study a possible system of notation.

The charge of the Subcommittee was to assess the extent to which the representational symbolism in use at that time (1990) in the field of $n$-dimensional crystallography may have become so nonuniform that it is unacceptably ambiguous. If the results of this assessment so warranted, the Subcommittee was charged further with proposing a set of recommendations for a unified nomenclature and symbolism for use in $n$-dimensional crystallography, following adequate discussion with other leaders in the field.

The first Report of the Subcommittee, presented herein, discusses the notation of point-group transfor- 
mations and geometric crystal classes. Further, a proposal is made for the standard setting of representative lattices for four-, five- and six-dimensional crystal families. The notation for arithmetic crystal classes, centring symbols, Bravais classes, and finally space groups in higher dimensions will be presented in a subsequent Report.

\section{Symbols and terms}

The theory of crystallographic point groups, lattices and space groups may be formulated quite independently of their possible applications by treating it as a mathematical problem. From this viewpoint, it becomes a matter of the determination of all non-isomorphic crystallographic space groups in $n$ dimensions. Applications enter in treating equivalence relations between groups or in introducing a nomenclature. Only 219 classes of space groups need be distinguished in three dimensions if any two space groups are regarded as equivalent if and only if they are isomorphic or, as shown by Bieberbach (1911), to be equivalent if they are conjugated subgroups of the general affine group. However, when the handedness of a structure is of importance, a finer classification becomes necessary, leading to the 230 classes because there are 11 three-dimensional enantiomorphic pairs. This extension is a well known example of the effect of physical considerations on the choice of the equivalence definitions. Inferences arising from applications will not be discussed in this Report. Instead, group-theoretical isomorphism will be used as an equivalence relation. The isomorphism classes of space groups lead naturally to arithmetic crystal classes and geometric crystal classes, which can be grouped into Bravais classes, systems and families.

This Report deals with symbols for orthogonal transformations, geometric crystal classes and with the choice of standard bases for lattices. The point groups in the same geometric crystal class, which are conjugate subgroups of the orthogonal group $O(n)$, receive the same symbol, and this is discussed in $\S 4$. In the same way, orthogonal transformations, which are elements of $O(n)$, receive the same symbol when they are conjugate in this group. This means that the symbol does not give information about the orientation of the orthogonal transformation. Sometimes, the term type of orthogonal transformation is used for this conjugacy class. Symbols for these transformations are treated in $\S 3$.

The geometric crystal classes can be grouped together in point-group systems and families, see $\$ 4$ and Table 3 . An explanation of various terms can be found in ch. 8 of Vol. A of ITC. The relation of the various terms used is indicated in Fig. 1.

A standard basis may be indicated for an invariant lattice in each family. Up to orientation, such a standard basis is characterized by its metric tensor with elements, $g_{i j}=\vec{a}_{i} \cdot \vec{a}_{j}$, where $\vec{a}_{i}(i=1, \ldots, n)$ are the basis vectors.
The standard bases are chosen to give a particularly simple form to the matrices of the point groups belonging to the family.

Some symbols used in this Report (the symbol as used in ITC is given in the second column):

\begin{tabular}{|c|c|c|}
\hline$n \mathrm{D}$ & & $n$-dimensional \\
\hline & $\mathbf{a}$ & Vector \\
\hline$\vec{b}$ & $(\mathbf{a}, \mathbf{b}), \mathbf{a} \cdot \mathbf{b}$ & $\underset{\vec{b}}{\text { Scalar product of vectors } \vec{a} \text { and }}$ \\
\hline$g_{i i}$ & & $\begin{array}{l}\text { Coefficients of the metric } \\
\text { tensor: } g_{i j}=\vec{a}_{i} \cdot \vec{a}_{j}\end{array}$ \\
\hline$(n)$ & $\mathfrak{E}$ & $n \mathrm{D}$ Euclidean group \\
\hline$(n)$ & & $\begin{array}{l}\text { Group of all } n \mathrm{D} \text { orthogonal } \\
\text { transformations }\end{array}$ \\
\hline (q) & & Euler function \\
\hline$\perp K_{2}$ & $\mathfrak{K}_{1} \perp \mathfrak{K}_{2}$ & External product (see $\S 4$ ) \\
\hline & & $\begin{array}{l}\text { Crystallographic orthogonal } \\
\text { transformation of order } q \\
\text { (see §3) }\end{array}$ \\
\hline
\end{tabular}

\section{Orthogonal transformations}

Point-group transformations are orthogonal transformations in $n \mathrm{D}$ space. They are represented by orthogonal matrices on an orthogonal basis. A point-group transformation is crystallographic if there is an $n \mathrm{D}$ lattice that is left invariant. On a basis of such an invariant lattice, the point-group transformation is represented by an integer matrix.

An orthogonal transformation can be put in matrix form, which is the direct sum of $1 \mathrm{D}$ and $2 \mathrm{D}$ orthogonal matrices, by a real basis transformation. Any other orthogonal transformation in the same conjugacy class of $O(n)$, i.e. of the same type, can be brought into the same block form. The $1 \mathrm{D}$ blocks are \pm 1 , the $2 \mathrm{D}$ blocks are of the form

$$
\left(\begin{array}{rr}
\cos \phi & -\sin \phi \\
\sin \phi & \cos \phi
\end{array}\right)
$$

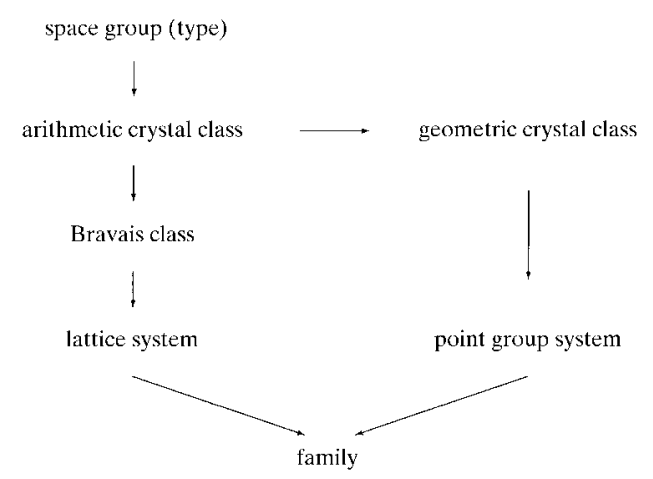

Fig. 1. The relation between various crystallographic equivalence concepts. 
If the transformation is of finite order $q$, the value of $\phi$ is $2 \pi p / q$, where $p$ and $q$ are mutually prime integers.

An integer matrix of finite order corresponds to an orthogonal transformation on the basis of an invariant lattice. Therefore, it can be brought into block form with blocks of dimension 1 or 2 by a real basis transformation. It can also be brought into block form by a rational basis transformation but the minimal dimension of the blocks is now generally larger. Rationally irreducible blocks can be written as a sum of blocks with eigenvalues $\exp (2 \pi i p / q)$ for fixed $q$ by a real transformation. The dimension of the rationally irreducible block is given by $\Phi(q)$, the Euler function; $\Phi(q)$ for integer $q$ is the number of integers coprime with $q$ and smaller than $q$.

This relationship suggests a possible notation. The type of orthogonal transformation of finite order is given by the rational numbers $p / q$ in the $2 \mathrm{D}$ blocks and the number of 1's and -1 's in the block form. These numbers are unique up to a permutation of blocks. All orthogonal transformations that can be transformed into each other by a real basis transformation give the same numbers. In particular, all elements of one conjugacy class in $O(n)$ are given the same characterizing numbers.

Considering the reduction by rational transformations, each rationally irreducible block of dimension higher than two can be reduced further to a sum of $2 \mathrm{D}$ blocks by a real transformation. In that case, the $\Phi(q)$ conjugate roots of unity occur simultaneously. They have the same $q$ value. This forms the basis of the proposal by Hermann (1949) for a notation of crystallographic transformations. His notation gives the sequence of orders of the rationally irreducible blocks. For example, the sequence 321 indicates a transformation which in reduced form is the direct sum of a $2 \mathrm{D}$ threefold rotation, a 1D inversion (of order 2) and the 1D identity. Therefore, such a sequence corresponds to a 4D transformation. Hermann denotes the eightfold rotation with values $p / q=1 / 8,3 / 8,5 / 8,7 / 8$, which is a $4 \mathrm{D}$ operation, by ' 8 '. This nomenclature is only applicable for crystallographic transformations and suppresses the information on the values of $p$. Therefore, we recommend a slightly different scheme.

The first principle is to use the same symbol for an orthogonal transformation in $n$ dimensions as that in $n+k$ dimensions obtained from the former by adding $k$ 1 's. The only exception is the unit element in $n$ dimensions denoted by $1_{n}$. The second principle is to retain the symbols in ITC in one, two and three dimensions, namely $1, m$ in one dimension, 2, 3, 4, 6 in two dimensions, and $\overline{1}, \overline{3}, \overline{4}, \overline{6}$ in three dimensions.

All information on the number of eigenvalues +1 is hence suppressed in the symbol, except in the case of the unit element $1_{n}$. If it is desired for some reason to indicate explicitly the dimension of the space in which a transformation acts, it is possible to add a number of digits 1 . Then, 411 is clearly a $4 \mathrm{D}$ transformation, to be distinguished from the 3D 41. If there are only eigenvalues +1 and exactly one eigenvalue -1 , then the symbol is $m$ according to the second principle above. Pairs of eigenvalues -1 can be combined to give 2D twofold rotations written as 2 . We call the number of eigenvalues different from unity the effective dimension of the orthogonal transformation.

A 3- (4- or 6-) fold rotation in two dimensions can be written as 3 ( 4 or 6 , respectively). The rotations 3 and $3^{2}$, as well as the pairs $4,4^{3}$ and $6,6^{5}$ denote the same rotation type because 3 and $3^{2}$ can be transformed into each other by a real transformation. This is generally true: orthogonal transformations $R$ and $R^{-1}$ have the same eigenvalues and, therefore, are of the same type.

A 5-, 8-, 10- or 12-fold rotation is not crystallographic in two dimensions (there is no invariant lattice). Moreover, there are two different rotations with the same order: 5 and $5^{2}$, which must be distinguished. The same holds for 8 and $8^{3}, 10$ and $10^{3}$, and for 12 and $12^{5}$.

There are three different choices for 7-, 9-, 14- or 18fold rotations in two dimensions: $7,7^{2}$ and $7^{3}$ and the triplets $9,9^{2}, 9^{4} ; 14,14^{3}, 14^{5} ; 18,18^{5}, 18^{7}$.

In general, for a $q$-fold rotation in two dimensions, there are $\Phi(q) / 2$ different rotations. For arbitrary dimensions, the rotation can be written as a sequence of numbers. For example, $75^{2} 3$ is equivalent to the direct sum of rotations $7,5^{2}$ and 3 . It exists in spaces of dimension 6 and higher and is of order 105. Its effective dimension is 6 and it can be written as

$\left(\begin{array}{cccccc}\cos (2 \pi / 7) & -\sin (2 \pi / 7) & 0 & 0 & 0 & 0 \\ \sin (2 \pi / 7) & \cos (2 \pi / 7) & 0 & 0 & 0 & 0 \\ 0 & 0 & \cos (4 \pi / 5) & -\sin (4 \pi / 5) & 0 & 0 \\ 0 & 0 & \sin (4 \pi / 5) & \cos (4 \pi / 5) & 0 & 0 \\ 0 & 0 & 0 & 0 & \cos (2 \pi / 3) & -\sin (2 \pi / 3) \\ 0 & 0 & 0 & 0 & \sin (2 \pi / 3) & \cos (2 \pi / 3)\end{array}\right)$

The same rotation in higher-dimensional spaces is denoted by the same symbol since the digit 1 is omitted. If $q$ becomes 10 or larger, it should be separated from the other digits by a thin space. If $q$ becomes 22 or larger, there can be confusion whether this is 22 or two digits 2 . Then one has to put the number in curly brackets: $\{22\}$ is a ten-dimensional rotation, but 22 is a four-dimensional rotation, usually denoted by $\overline{1_{4}}$.

In the case of a crystallographic transformation, an eigenvalue $\exp (2 \pi i p / q)$ is always accompanied by its conjugates, the $\Phi(q)$ eigenvalues $\exp \left(2 \pi i p^{\prime} / q\right)$ with $p^{\prime}$ coprime with $q$. This allows the symbol for crystallographic transformations of dimension 4 or higher to be shortened. For example, the rotation $77^{2} 7^{3}$ is crystallographic and minimally of dimension 6 . Because the six eigenvalues always come together, it is sufficient to give only one of them (with the smallest denominator value). The symbol is chosen as [7], standing for the longer symbol $77^{2} 7^{3}$. This allows the crystallographic transformation [5] $=55^{2}$ to be distinguished from the noncrystallographic transformation 55, another 4D transformation. 
A transformation with determinant -1 contains an odd number of eigenvalues -1 . It is customary in three dimensions to denote the product of the inversion and a $q$-fold rotation $(q>2)$ by an overbar: $\overline{4}$ is a 3D transformation obtained as the product of $\overline{1}$ and the rotation 4. Its matrix is the direct sum of the $2 \mathrm{D}$ matrix $4^{3}$ (which is of the same type as 4) and the number -1 .

$$
\overline{4}=-\left(\begin{array}{rrr}
0 & -1 & 0 \\
1 & 0 & 0 \\
0 & 0 & 1
\end{array}\right)=\left(\begin{array}{rrr}
0 & 1 & 0 \\
-1 & 0 & 0 \\
0 & 0 & -1
\end{array}\right) .
$$

An exception is the product of a twofold rotation and $\overline{1}$, because this is a mirror, denoted by $m$, not by $\overline{2}$. It should be noted that the transformation with determinant -1 denoted by $\overline{3}$ is the product of $\overline{1}$ and the rotation 3 , but at the same time it is the sum of the rotation $6^{5}$ and -1 . On the other hand, $\overline{6}$ is the sum of $3^{2}$ and -1 . (In ITC, the corresponding group $\overline{6}$ is the same as $3 / \mathrm{m}$.) In the past, one has chosen a symbol using multiplication $(\overline{6}=\overline{1} \times 6)$ rather than summation $(3 / m=3+m)$.

$$
\begin{aligned}
\overline{6} & =-\left(\begin{array}{ccc}
\cos (2 \pi / 6) & -\sin (2 \pi / 6) & 0 \\
\sin (2 \pi / 6) & \cos (2 \pi / 6) & 0 \\
0 & 0 & 1
\end{array}\right) \\
& =\left(\begin{array}{ccr}
\cos (4 \pi / 3) & -\sin (4 \pi / 3) & 0 \\
\sin (4 \pi / 3) & \cos (4 \pi / 3) & 0 \\
0 & 0 & -1
\end{array}\right) ; \\
\overline{3} & =\left(\begin{array}{ccr}
\cos (10 \pi / 6) & -\sin (10 \pi / 6) & 0 \\
\sin (10 \pi / 6) & \cos (10 \pi / 6) & 0 \\
0 & 0 & -1
\end{array}\right) .
\end{aligned}
$$

This notation is not different in higher dimensions: an odd-dimensional transformation with determinant -1 is the product of a rotation and the inversion in the odddimensional space and can be denoted by a bar above the digits corresponding to the $q$-fold rotation $(q>2)$.

$\overline{55^{2}}=\left(\begin{array}{cc}\cos (14 \pi / 10) & -\sin (14 \pi / 10) \\ \sin (14 \pi / 10) & \cos (14 \pi / 10) \\ 0 & 0 \\ 0 & 0 \\ 0 & 0\end{array}\right.$

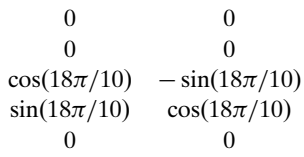

$\left.\begin{array}{r}0 \\ 0 \\ 0 \\ 0 \\ -1\end{array}\right)$

This transformation is of order 10 . The same symbol can be used for a $(5+k)$-dimensional transformation, obtainable from this 5D operation by adding $k$ diagonal terms 1 . This is equivalent to obtaining $\overline{55^{2}}$ from the rotation $55^{2}$ on multiplication by the total inversion in a space of dimension equal to the effective dimension of $55^{2}$ (which is four) plus one.

A general $n \mathrm{D}$ rotation (with determinant +1 ) is denoted by a series $q_{1}^{p_{1}} q_{2}^{p_{2}} \ldots 22 \ldots$ of $k$ digits. This is the symbol already in use for a $2 k$-dimensional rotation. The number of omitted digits 1 is $n-2 k$. The symbol for a general orthogonal transformation of determinant -1 is

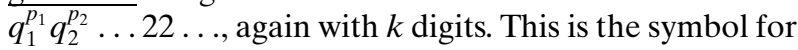

a $(2 k+1)$-dimensional operation. In $n \mathrm{D}$ space, there are still $n-2 k-1$ omitted digits 1 .

Inversion is a special case. It is either a rotation (in even dimensions) or has a determinant equal to -1 (in odd dimensions). For even dimensions, this element may be written as a series of 2's. To stress its special role, the transformation in $n$ dimensions is denoted as $\overline{1_{n}}$.

A consequence of this system of notation is that a property sometimes used in three dimensions is not generally true: if a $q$-fold rotation belongs to the Laue group, then the transformation $\bar{q}$ also belongs to it. Indeed it is true that, if $A$ is a rotation without twofold component and of dimension $2 k$, then $\bar{A}$ is a $(2 k+1)$-dimensional orthogonal transformation and $-\bar{A}=\overline{1_{2 k+1}} \times \bar{A}=A$. In particular, $-\overline{3}=3$ in three dimensions, of course. However, this is no longer true in an arbitrary dimension. In general, $q$ is not equal to $-\bar{q}$. In four dimensions, the transformation 4 is actually 411 . Then its opposite $-411=42$ is also a rotation and belongs to the same Laue group as 4 . But $\overline{4}$ in four dimensions is actually $\overline{4} 1$ and has determinant -1 , which cannot be changed by multiplication with $\overline{1_{4}}$. The 4D operators 411 and $\overline{4} 1$, in short notation 4 and $\overline{4}$, leave different lattices invariant. It should be noted that one can make the remark already in two dimensions. There, -4 is not $\overline{4}$ but 4 itself.

It is noteworthy that, in some cases, the cyclic groups generated by $q$ and $\bar{q}$ (for integer $q$ ) belong to the same crystal family (family preserving $\bar{q}$ ) and, in other cases, to different families (family breaking $\bar{q}$ ). Wondratschek (1998) observed that, in odd dimensions, the groups generated by $q$ and $\bar{q}$ belong to the same crystal family if and only if there is exactly one constituent +1 (and no constituent -1 ) in the operation $q$ and, hence, one -1 and no +1 in $\bar{q}$. In all other cases, $\bar{q}$ is family breaking.

The Subcommittee has discussed this point extensively. The reasons for recommending the use of the overbar in this Report are, in short, the following.

(i) The notation with a bar is a natural generalization of that in three dimensions.

(ii) If a transformation $T$ leaves the Fourier intensity the same, then $-T$ has this property also. It is very simple to determine the symbol for $-T$ if one knows that for $T$, but that is not necessarily $\bar{T}$.

(iii) All discussed alternatives were more complicated. The reason $\overline{6}$ was chosen in three dimensions and not $3 / m$ is that the former symbol is simpler.

(iv) It is always possible to use a more extensive symbol: then -41 is $\overline{4}$.

Examples (notice that rotations over angles $\phi$ and $-\phi$ have the same symbol):

$$
[5]=\left(\begin{array}{cccc}
\cos (2 \pi / 5) & -\sin (2 \pi / 5) & 0 & 0 \\
\sin (2 \pi / 5) & \cos (2 \pi / 5) & 0 & 0 \\
0 & 0 & \cos (4 \pi / 5) & -\sin (4 \pi / 5) \\
0 & 0 & \sin (4 \pi / 5) & \cos (4 \pi / 5)
\end{array}\right)
$$




$$
\overline{43}=\left(\begin{array}{ccccr}
\cos (2 \pi / 4) & -\sin (2 \pi / 4) & 0 & 0 & 0 \\
\sin (2 \pi / 4) & \cos (2 \pi / 4) & 0 & 0 & 0 \\
0 & 0 & \cos (2 \pi / 6) & -\sin (2 \pi / 6) & 0 \\
0 & 0 & \sin (2 \pi / 6) & \cos (2 \pi / 6) & 0 \\
0 & 0 & 0 & 0 & -1
\end{array}\right) ;
$$

$$
\overline{43} 2=\left(\begin{array}{ccccrrr}
\cos (2 \pi / 4) & -\sin (2 \pi / 4) & 0 & 0 & 0 & 0 & 0 \\
\sin (2 \pi / 4) & \cos (2 \pi / 4) & 0 & 0 & 0 & 0 & 0 \\
0 & 0 & \cos (2 \pi / 6) & -\sin (2 \pi / 6) & 0 & 0 & 0 \\
0 & 0 & \sin (2 \pi / 6) & \cos (2 \pi / 6) & 0 & 0 & 0 \\
0 & 0 & 0 & 0 & -1 & 0 & 0 \\
0 & 0 & 0 & 0 & 0 & -1 & 0 \\
0 & 0 & 0 & 0 & 0 & 0 & -1
\end{array}\right) .
$$

Notice that the 4D transformation 32 and the $6 \mathrm{D}$ transformations 422,432 and 622 have the same symbols as the corresponding 3D point groups in the standard notation. It should be clear from the context what kind of object one considers.

Recommendation 1. An orthogonal transformation of finite order that can be written after a suitable real basis transformation as the direct sum

$$
\begin{gathered}
S=\bigoplus_{i=1}^{r}\left(\begin{array}{cc}
\cos 2 \pi p_{i} / q_{i} & -\sin 2 \pi p_{i} / q_{i} \\
\sin 2 \pi p_{i} / q_{i} & \cos 2 \pi p_{i} / q_{i}
\end{array}\right) \oplus \bigoplus_{j=1}^{m} a_{j} \oplus \bigoplus_{k=1}^{l} b_{k}, \\
a_{j}=-1, b_{k}=1,2 r+m+l=n, \quad \text {, }
\end{gathered}
$$

should be written in full as

$$
q_{1}^{p_{1}} q_{2}^{p_{2}} \ldots 22 \ldots
$$

with $m / 2$ 's when $m$ is even,

$$
\overline{d_{1}^{c_{1}} d_{2}^{c_{2}} \ldots} 22 \ldots
$$

with $(m-1) / 2$ 2's when $m$ is odd, and with $c_{i} / d_{i}=p_{i} / q_{i}+\frac{1}{2}(\bmod 1)$, but it is

$$
1_{n}
$$

when $l=n$ (only eigenvalues +1$)$, and

$$
\overline{1_{m}},
$$

when $m=n$ (only eigenvalues -1 ). The order is such that

$$
q_{i} \geq q_{i+1} \geq \ldots, \quad p_{i} \leq p_{i+1} \quad \text { if } q_{i}=q_{i+1} .
$$

Recommendation 2. For consistency with current practice, $m$ should be used for $\overline{1_{1}}, 2$ is used exceptionally instead of $\overline{1_{2}}$, and $\overline{1}$ is used instead of $\overline{1_{3}}$.

Recommendation 3 . In the case of a crystallographic orthogonal transformation, a short form is obtained by combining conjugate blocks with the same $q$ into the symbol $[q]$. This represents the sequence

$$
[q]=q^{p_{1}} \ldots q^{p_{e}}
$$

where $p_{1}, \ldots, p_{e}$ are the $\Phi(q) / 2$ integers that are coprime with $q$ and smaller than $q / 2$.

Recommendation 4. The symbol does not depend on the number of eigenvalues +1 . However, if there are only eigenvalues +1 and no others, then the transformation should be denoted by $1_{n}$. If it is desirable to indicate explicitly the dimension of the transformation, as many digits 1 may be added as there are eigenvalues equal to +1 .

The symbols for elements of crystallographic point groups in dimensions up to 6 are given in Table 1 . The sequence mentioned in the heading is the Hermann symbol.

\section{Geometric crystal classes}

An $n \mathrm{D}$ point group is a subgroup of the orthogonal group in $n$ dimensions. If it leaves a subspace invariant, it can, by a suitable real basis transformation, be put into reduced form, i.e. all elements are simultaneously in the form of direct sums of blocks having the same dimension for all elements. If the dimensions of the invariant subspaces are $n_{1}, n_{2}, \ldots$, then the point group is said to be $\left(n_{1}+n_{2}+\ldots\right)$-reducible or $\left(n_{1}+n_{2}+\ldots\right)$ - $R$-reducible. All point groups in the same geometric crystal class have the same reducibility character. As in the case of point transformations, point groups are denoted by a symbol for the space in which they act effectively. In particular, the symbols in two and three dimensions should remain the same as those used in ITC. These symbols remain the symbols for the geometric crystal class. For higher-dimensional spaces, the symbols can, to a large extent, be based on the reducibility of the point group, just as for point transformations. Some of these ideas follow those of Weigel et al. $(1987,1993)$, who have proposed symbols for point groups in four, five and six dimensions.

A special case for a point group occurs in reduced form with $m$ blocks if it is the direct product of $m$ subgroups consisting of unit matrices, except for one of the $m$ blocks. An example is the 3D group 4/mmm with generating matrices

$$
\left(\begin{array}{rrr}
0 & -1 & 0 \\
1 & 0 & 0 \\
0 & 0 & 1
\end{array}\right), \quad\left(\begin{array}{rrr}
-1 & 0 & 0 \\
0 & 1 & 0 \\
0 & 0 & 1
\end{array}\right), \quad\left(\begin{array}{rrr}
1 & 0 & 0 \\
0 & 1 & 0 \\
0 & 0 & -1
\end{array}\right) .
$$

This is the direct product of the subgroup $4 \mathrm{~mm}$ of order eight generated by the first two matrices and the group $m$ of order two generated by the third matrix. In this situation, the group is the direct product of subgroups acting only in one of the $m$ mutually orthogonal invariant subspaces. The symbol for such a group can be related to those for lower-dimensional point groups:

$$
K=K_{1} \perp K_{2} \perp \ldots \perp K_{m} .
$$

In the example, group $4 / \mathrm{mmm}$ would have the alternative symbol $4 m m \perp m$. However, preserving the principle that standard notation be retained in dimensions up to three, the preferred symbol remains $4 / \mathrm{mmm}$. 
Table 1. Symbols for crystallographic point-group transformations

\begin{tabular}{|c|c|c|c|c|c|c|c|}
\hline $\begin{array}{l}\text { Hermann (1949) } \\
\text { symbol }\end{array}$ & Order & Parity & Symbol & $\begin{array}{l}\text { Hermann (1949) } \\
\text { symbol }\end{array}$ & Order & Parity & Symbol \\
\hline$\geq 1$ & 1 & + & 1 & 2 & 2 & - & $m$ \\
\hline 22 & 2 & + & 2 & 3 & 3 & + & 3 \\
\hline 4 & 4 & + & 4 & 6 & 6 & + & 6 \\
\hline 222 & 2 & - & $\overline{1}$ & 32 & 6 & - & $\overline{6}$ \\
\hline 42 & 4 & - & $\overline{4}$ & 62 & 6 & - & $\overline{3}$ \\
\hline 2222 & 2 & + & $\overline{1}_{4}$ & 322 & 6 & + & 32 \\
\hline 33 & 3 & + & 33 & 422 & 4 & + & 42 \\
\hline 43 & 12 & + & 43 & 44 & 4 & + & 44 \\
\hline 5 & 5 & + & [5] & 622 & 6 & + & 62 \\
\hline 63 & 6 & + & 63 & 64 & 12 & + & 64 \\
\hline 66 & 6 & + & 66 & 8 & 8 & + & [8] \\
\hline (10) & 10 & + & [10] & (12) & 12 & + & [12] \\
\hline 22222 & 2 & - & $\overline{1}_{5}$ & 3222 & 6 & - & $\overline{6} 2$ \\
\hline 332 & 6 & - & $\frac{5}{66}$ & 4222 & 4 & - & $\overline{4} 2$ \\
\hline 432 & 12 & - & $\overline{64}$ & 442 & 4 & - & $\overline{44}$ \\
\hline 52 & 10 & - & $\overline{[10]}]$ & 6222 & 6 & - & 32 \\
\hline 632 & 6 & - & $\frac{15}{63}$ & 642 & 12 & - & 43 \\
\hline 662 & 6 & - & $\frac{5}{33}$ & 82 & 8 & - & {$[8]$} \\
\hline 102 & 10 & - & [5] & 122 & 12 & - & {$[12]$} \\
\hline 222222 & 2 & + & $\overline{1}_{6}$ & 32222 & 6 & + & 322 \\
\hline 3322 & 6 & + & $\begin{array}{l}26 \\
332\end{array}$ & 333 & 3 & + & 333 \\
\hline 42222 & 4 & + & 422 & 4322 & 12 & + & 432 \\
\hline 433 & 12 & + & 433 & 4422 & 4 & + & 442 \\
\hline 443 & 12 & + & 443 & 444 & 4 & + & 444 \\
\hline 522 & 10 & + & {$[5] 2$} & 53 & 15 & + & {$[5] 3$} \\
\hline 54 & 20 & + & {$[5] 4$} & 62222 & 6 & + & 622 \\
\hline 6322 & 6 & + & 632 & 633 & 6 & + & 633 \\
\hline 6422 & 12 & + & 642 & 643 & 12 & + & 643 \\
\hline 644 & 12 & + & 644 & 65 & 30 & + & $6[5]$ \\
\hline 6622 & 6 & + & 662 & 663 & 6 & + & 663 \\
\hline 664 & 12 & + & 664 & 666 & 6 & + & 666 \\
\hline 7 & 7 & + & [7] & 822 & 8 & + & {$[8] 2$} \\
\hline 83 & 24 & + & {$[8] 3$} & 84 & 8 & + & {$[8]^{4}$} \\
\hline 86 & 24 & + & {$[8] 6$} & 9 & 9 & + & [9] \\
\hline 1022 & 10 & + & {$[10] 2$} & 103 & 30 & + & {$[10] 3$} \\
\hline 104 & 20 & + & {$[10] 4$} & 106 & 30 & + & {$[10] 6$} \\
\hline 1222 & 12 & + & {$[12] 2$} & 123 & 12 & + & {$[12] 3$} \\
\hline 124 & 12 & + & {$[12] 4$} & 126 & 12 & + & {$[12] 6$} \\
\hline 14 & 14 & + & [14] & 18 & 18 & + & [18] \\
\hline
\end{tabular}

$[5]=55^{2},[8]=88^{3},[10]=1010^{3},[12]=1212^{5},[7]=77^{2} 7^{3},[9]=99^{2} 9^{4},[14]=1414^{3} 14^{5},[18]=1818^{5} 18^{7}$. Parity is + if the determinant is positive, otherwise -.

Choosing a basis of an invariant lattice that makes the simple structure evident is very useful because the matrices remain simple.

An $\left(m_{1}+m_{2}\right)$-reducible point group $H$ is always a subgroup of an $\left(m_{1}+m_{2}\right)$-dimensional point group $K=K_{1} \perp K_{2}$, where $K_{i}$ is an $m_{i}$-dimensional point group. The elements $h$ of $H$ can be written as pairs $\left(h_{1}, h_{2}\right)$ of elements of $K_{1}$ and $K_{2}$, respectively. The elements $h_{1}$ generate $K_{1}$ and the elements $h_{2}$ generate $K_{2}$. $H$ is then said to be a subdirect product of $K_{1}$ and $K_{2}$.

If the elements of the subdirect product of $K_{1}$ and $K_{2}$ are denoted by $\left(h_{1}, h_{2}\right)$, then the elements $\left(h_{1}, e\right)$ form an invariant subgroup $H_{1}$ and the elements $\left(e, h_{2}\right)$ an invariant subgroup $H_{2}$ of $K_{1}$ and $K_{2}$, respectively. The quotient groups $K_{1} / H_{1}$ and $K_{2} / H_{2}$ are isomorphic. All subdirect products of $K_{1}$ and $K_{2}$ are obtained by considering all invariant subgroups $H_{1}$ and $H_{2}$, taking those pairs for which $K_{1} / H_{1}$ and $K_{2} / H_{2}$ are isomorphic and considering all isomorphisms from one to the other.

A special case of a subdirect product is that in which $K_{1}=H_{1}$ and $K_{2}=H_{2}$. In this case, the subdirect product is simply a direct product of $H_{1}$ and $H_{2}$, and is denoted by $H_{1} \perp H_{2}$.

Suppose a group is the subdirect product of an $n$-dimensional point group $K_{1}$ and an $m$-dimensional point group $K_{2}$. The elements are pairs $\left(h_{1}, h_{2}\right)$, where 
Table 2. Geometric crystal classes in one dimension, two dimensions and three dimensions

\begin{tabular}{|c|c|c|c|c|c|c|}
\hline $\begin{array}{l}\text { Point-group } \\
\text { system }\end{array}$ & Order & Symbol & Alternative & Order & Symbol & Alternative \\
\hline 1D_1 & 1 & 1 & & 2 & $m$ & \\
\hline 2D_1 & 1 & 1 & $1_{2}$ & 2 & 2 & \\
\hline 2D_2 & 2 & $m$ & & 4 & $\mathrm{~mm}$ & $m \perp m$ \\
\hline 2D_3 & 4 & 4 & & 8 & $4 m m$ & \\
\hline \multirow[t]{2}{*}{ 2D_4 } & 3 & 3 & & 6 & $3 m$ & \\
\hline & 6 & 6 & & 12 & $6 \mathrm{~mm}$ & \\
\hline 3D_1_1 & 1 & 1 & $1_{3}$ & 2 & $\overline{1}$ & $\overline{1_{3}}$ \\
\hline \multirow[t]{2}{*}{ 3D_2_1 } & 2 & $m$ & & 2 & 2 & \\
\hline & 4 & $2 / m$ & $2 \perp m$ & & & \\
\hline \multirow[t]{2}{*}{ 3D_3_1 } & 4 & 222 & $m m(m m)$ & 4 & $m m 2$ & $m \perp m$ \\
\hline & 8 & $\mathrm{mmm}$ & $m \perp m \perp m$ & & & \\
\hline \multirow{4}{*}{ 3D_4_1 } & 4 & 4 & & 8 & $4 m m$ & \\
\hline & 4 & $\overline{4}$ & $4(m)$ & 8 & $4 / m$ & $4 \perp m$ \\
\hline & 8 & 422 & $4 m(1 m)$ & 8 & $42 m$ & $4 m(\mathrm{~mm})$ \\
\hline & 16 & $4 / \mathrm{mmm}$ & $4 m m \perp m$ & & & \\
\hline \multirow[t]{3}{*}{ 3D_5_1 } & 3 & $\underline{3}$ & & 6 & $3 m$ & \\
\hline & 6 & $\overline{3}$ & $3 \times \overline{1}$ & 6 & 32 & $3 m(1 m)$ \\
\hline & 12 & $\overline{3} m$ & $3 m \times \overline{1}$ & & & \\
\hline \multirow[t]{4}{*}{ 3D_5_2 } & 6 & 6 & & 12 & $6 \mathrm{~mm}$ & \\
\hline & 6 & $\overline{6}$ & $3 \perp m$ & 12 & $6 / m$ & $6 \perp m$ \\
\hline & 12 & $\overline{6} 2 m$ & $3 m \perp m$ & 12 & 622 & $6 m(1 m)$ \\
\hline & 24 & $6 / \mathrm{mmm}$ & $6 m m \perp m$ & & & \\
\hline \multirow{3}{*}{ 3D_6_1 } & 12 & 23 & & 24 & $m \overline{3}$ & $23 \times \overline{1}$ \\
\hline & 24 & 432 & & 24 & $\overline{4} 3 m$ & \\
\hline & 48 & $m \overline{3} m$ & $432 \times \overline{1}$ & & & \\
\hline
\end{tabular}

the elements $h_{1}$ form the group $K_{1}$ and the elements $h_{2}$ the group $K_{2}$. To each element of point group $K_{1}$, there corresponds at least one element of $K_{2}$ and vice versa. Its symbol may be taken as that of $K_{1}$ with corresponding elements of $K_{2}$ placed between parentheses. For example, the subgroup of $4 m m \perp m$ generated by the pairs $(4, m)$ and $(m, 1)$ is the subdirect product of $4 m m$ and $m$ and can be denoted by $4 m m(m 1 m)$. The second $m$ in $4 \mathrm{~mm}$ is the product of 4 and the first $\mathrm{m}$. Correspondingly, the second $m$ in the parentheses is the product of $m$ and 1. Because the symbols for subdirect products tend to become rather long, one writes preferentially $4 m(m 1)$ instead of $4 m m(m 1 m)$. If the subgroup $H_{1}$ of the elements of $K_{1}$ associated with the identity in $K_{2}$ is not trivial, then the corresponding symbols in parentheses are 1's and analogously for $\mathrm{H}_{2}$. The example $4 / \mathrm{mmm}$ could be written as $4 \mathrm{~mm} 1(111 \mathrm{~m})$, but in this case the shorter notation is $4 m m \perp m$.

The subgroups 4 and $4 m m$ of the $3 \mathrm{D}$ group $4 m m \perp m$ are $2 \mathrm{D}$ and are denoted by 4 and $4 \mathrm{~mm}$, respectively. The subgroup $4 / m$ has the same reducibility character as $4 / \mathrm{mmm}$ and is written as $4 \perp m$. The other subgroups are more complicated. For the group 422, the elements in the first 2D block form the group $4 \mathrm{~mm}$ but the three elements indicated (4,2 and 2) are associated with 1 , -1 and -1 , respectively. The symbol is therefore $4 \mathrm{~mm}(1 \mathrm{~mm})$. The group is generated by

$$
\left(\begin{array}{rrr}
0 & -1 & 0 \\
1 & 0 & 0 \\
0 & 0 & 1
\end{array}\right), \quad\left(\begin{array}{rrr}
-1 & 0 & 0 \\
0 & 1 & 0 \\
0 & 0 & -1
\end{array}\right) .
$$

Similarly, the group $\overline{4} 2 m$ may be written as $4 m m(m m 1)$. Generators are

$$
\left(\begin{array}{rrr}
0 & -1 & 0 \\
1 & 0 & 0 \\
0 & 0 & -1
\end{array}\right), \quad\left(\begin{array}{rrr}
-1 & 0 & 0 \\
0 & 1 & 0 \\
0 & 0 & -1
\end{array}\right) .
$$

However, because the notation for three-dimensional point groups should be retained, this procedure is only used in higher dimensions and 422 is written instead of $4 m m(1 \mathrm{~mm})$ and $\overline{4} 2 \mathrm{~m}$ instead of $4 \mathrm{~mm}(\mathrm{~mm} 1)$.

The symbols for one-, two- and three-dimensional groups are given in Table 2, both with their current ITC symbol and according to the rules of higherdimensional crystallography. The symbols for the $R$ reducible $4 \mathrm{D}$ point groups are given in Table 3 . They are $(3+1)-,(2+2)-,(2+1+1)-$ or $(1+1+1+1)$-reducible. The geometric crystal classes are combined into point-group systems, the ordering of which is discussed in the next section.

More than one symbol is occasionally proposed because there are arguments in favour of various options, just as in three dimensions $\overline{6}$ has been chosen instead of $3 / \mathrm{m}$, although there are good arguments for 
Table 3. (R-reducible) geometric crystal classes in four dimensions

\begin{tabular}{|c|c|c|c|c|c|c|}
\hline $\begin{array}{l}\text { Point-group } \\
\text { system }\end{array}$ & Order & Symbol & Alternative & Order & Symbol & Alternative \\
\hline 01_1 & $\begin{array}{l}1 \\
2\end{array}$ & $\frac{1}{1_{4}}$ & & & & \\
\hline 02_1 & $\begin{array}{l}2 \\
4\end{array}$ & $\stackrel{m}{\overline{1} \perp m}$ & & 2 & $\overline{1}$ & $\overline{1_{3}}$ \\
\hline 03_1 & $\begin{array}{l}2 \\
4\end{array}$ & $\begin{array}{l}2 \\
2 \perp 2\end{array}$ & & & & \\
\hline 04_1 & $\begin{array}{l}4 \\
4 \\
8\end{array}$ & $\begin{array}{l}2 m m \\
21(m 2) \\
2 \perp m m\end{array}$ & $\begin{array}{l}m \perp m \\
\overline{1} . \overline{1} \\
2 \perp m \perp m\end{array}$ & 4 & $2 \perp m$ & $2 / m$ \\
\hline 05_1 & 4 & $4(4)$ & 44 & & & \\
\hline 06_1 & $\begin{array}{l}3 \\
6\end{array}$ & $\begin{array}{l}3(3) \\
6(6)\end{array}$ & $\begin{array}{l}33 \\
66\end{array}$ & & & \\
\hline 07_1 & $\begin{array}{l}4 \\
8\end{array}$ & $222 \times \overline{1_{4}}$ & & & & \\
\hline $07 \_2$ & $\begin{array}{r}8 \\
8 \\
16\end{array}$ & $\begin{array}{l}\mathrm{mmm} \\
222 \perp m \\
\mathrm{mmmm}\end{array}$ & $\begin{array}{l}m \perp m \perp m \\
m \perp m \perp m \perp m\end{array}$ & & & \\
\hline 08_1 & $\begin{array}{r}4 \\
8 \\
8 \\
16\end{array}$ & $\begin{array}{l}4 \\
4 m m \\
4 m(12) \\
4 m m \perp 2\end{array}$ & $4 . \overline{1}$ & $\begin{array}{l}4 \\
8 \\
8\end{array}$ & $\begin{array}{l}4(2) \\
4 \perp 2 \\
4 m(22)\end{array}$ & $\begin{array}{l}42 \\
42 . \overline{1}\end{array}$ \\
\hline 09_1 & $\begin{array}{r}3 \\
6 \\
6 \\
12\end{array}$ & $\begin{array}{l}3 \\
3 m \\
32(1 m) \\
\overline{3} m(m 1)\end{array}$ & $\begin{array}{l}3 . \overline{1} \\
3 m \times \overline{1_{4}}\end{array}$ & 6 & $\overline{3}(m)$ & 62 \\
\hline 09_2 & $\begin{array}{r}6 \\
12 \\
12 \\
24\end{array}$ & $\begin{array}{l}6 \\
6 \mathrm{~mm} \\
3 m \perp 2 \\
6 m m \perp 2\end{array}$ & & $\begin{array}{r}6 \\
12 \\
12\end{array}$ & $\begin{array}{l}3 \perp 2 \\
6 \perp 2 \\
6 m(12)\end{array}$ & $6 . \overline{1}$ \\
\hline 10_1 & 8 & $4 m(4 m)$ & 44.2 & & & \\
\hline $\begin{array}{l}11 \_1 \\
12 \_1\end{array}$ & $\begin{array}{r}6 \\
12 \\
4\end{array}$ & $\begin{array}{l}3 m(3 m) \\
6 m(6 m)\end{array}$ & $\begin{array}{l}33.2 \\
66.2\end{array}$ & & & \\
\hline $12 z_{-}$ & $\begin{array}{r}4 \\
8 \\
8 \\
16\end{array}$ & $\begin{array}{l}\frac{4}{4} \times \overline{1_{4}} \\
\overline{4} 2 m \\
\overline{4} 2 m \times \overline{1_{4}}\end{array}$ & & 8 & $4 m(m 2)$ & $\overline{4} . \overline{1}$ \\
\hline $12 \_2$ & $\begin{array}{r}8 \\
8 \\
16 \\
16 \\
16 \\
32\end{array}$ & $\begin{array}{l}4 \perp m \\
422 \\
4 m m \perp m \\
422 \perp m \\
422 \times \overline{1_{4}} \\
4 m m \perp m m\end{array}$ & $4 / \mathrm{mmm}$ & $\begin{array}{r}8 \\
8 \\
16 \\
16\end{array}$ & $\begin{array}{l}\overline{4} \perp m \\
4 m(2 m) \\
4 \perp m m \\
\overline{4} 2 m \perp m\end{array}$ & 42.2 \\
\hline 13_1 & $\begin{array}{r}6 \\
6 \\
12 \\
12 \\
12 \\
24\end{array}$ & $\begin{array}{l}\overline{3} \\
3 \perp m \\
\overline{3} m \\
32 \perp m \\
\overline{3} m(m m)\end{array}$ & 62.2 & $\begin{array}{r}6 \\
12 \\
12 \\
12\end{array}$ & $\begin{array}{l}32 \\
\overline{3} \perp m \\
\overline{3} m(1 m) \\
3 m \perp m\end{array}$ & $\begin{array}{l}\overline{3} . \overline{1} \\
\overline{6} 2 \mathrm{~m}\end{array}$ \\
\hline
\end{tabular}


Table 3 (cont.)

Point-group system

13_2

15_1

16_1

17_1

18_1

17_2

19_1

19_2

20_1

Symbol
$6 \perp m$
622
$61(m 2)$
$6 \perp m m$
$62 m \perp m$
$6 m 1(1 m 2)$
$6 m m \perp m m$

$8\left(8^{3}\right)$

$8 m\left(8^{3} m\right)$

$5\left(5^{2}\right)$

$5 m\left(5^{2} m\right)$

$10 m\left(10^{3} m\right)$

$12\left(12^{5}\right)$

$12 m\left(12^{5} m\right)$

41(42)

$4 m 1(412)$

$4 m 1(m 42)$

$4 m 11(41 \mathrm{~mm})$

$4 \perp 4$

$4 m 1(1 m 4)$

$4 m m \perp 4$

$4 m m \perp 4 m m$

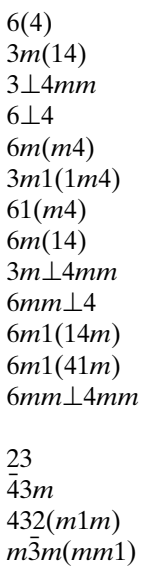

$m \overline{3}$

432

$432 \perp m$

$m \overline{3} \mathrm{~m}(\mathrm{mmm})$

$m \overline{3} m \perp m$

6(3)

61(32)

$6 m 1(3 m 2)$

Alternative
$6 / m$
$\overline{3} \times 2$
$3 m \perp m m$
$622 \times \overline{1_{4}}$

[8]

[8].2

[5]

[5].2

[10].2

[12]

[12].2

$44 \times 2$

44. $\mathrm{mm}$

$\overline{4} . \overline{4}$

44. $\mathrm{mmm}$

422.4

64

$3 . \overline{4}$

$\overline{3} . \overline{4}$

$\overline{3} . \underline{4}$

$6 . \overline{4}$

64. $\mathrm{mm}$

24

$\overline{4} 3 m \times \overline{1_{4}}$

$432 \times \overline{1_{4}}$

63

$63 \times \overline{1_{4}}$

$63.2 \times \overline{1_{4}}$

10

16

Ord
12
12
24
24
24

$10\left(10^{3}\right)$

Symbol

$3 \perp \mathrm{mm}$

$3 m(2 m)$

$622 \perp m$

$6 \mathrm{~mm} \perp \mathrm{m}$

$6 m 1(m 12)$

\section{$6 / \mathrm{mmm}$}

$\overline{3} m \times 2$

$\overline{6} \perp m$

32.2

[10]

$4 m 1(4 m 2)$

44.222

$4 m 1(142)$

$4 m 1(m 14)$

$4 . \overline{4}$

$\overline{4} 2 m .4$

$3 \perp 4$

$3 m \perp 4$

$6 m(4 m)$

$3 m 11(14 m m)$

61 $(4 m)$

$6 m 1(412)$

64.2

$\overline{6} . \overline{4}$

64. $m$

$3 m \times 42$

$6 \perp 4 m m$

$6 m 1(1 m 4)$

$6 m 1(m 14)$

$m \overline{3}(\mathrm{~mm})$

$23 \times \overline{1_{4}}$

$23 \perp m$

$\overline{4} 3 m(\mathrm{~m} 1 \mathrm{~m})$

$m \overline{4} 3 m \perp m$

$\overline{4} 3 m \perp m$

$m \overline{3} m(11 m)$

$6 m(3 m)$

63.2 
Table 3 (cont.)

Point-group

system

20_2

20_3

$\begin{array}{cl}\text { Order } & \text { Symbol } \\ 9 & 3 \perp 3 \\ 18 & 61(63) \\ 18 & 61(m 3) \\ 36 & 6 m 1(613) \\ 36 & 3 m \perp 3 m \\ 36 & 6 m 1(m 63) \\ 72 & 6 m 11(613 m) \\ & \\ 18 & 6 \perp 3 \\ 36 & 6 m m \perp 3 \\ 36 & 6 \perp 6 \\ 36 & 6 m 1(163) \\ 72 & 6 m m \perp 3 m \\ 72 & 6 m 1(1 m 6) \\ 144 & 6 m m \perp 6 m m\end{array}$

Alternative

$66 \times 3$

$\overline{3} .3$
Order

18

18

36

36
36

36
36
72
72

Symbol

$3 m \perp 3$

$3 m 1(1 m 3)$

$6 m 1(6 m 3)$

$6 m 1(m 13)$
$6 \perp 3 m$

$6 m 1(1 m 3)$

$6 m m \perp 6$

$6 m 11(163 m)$ both. It should become clear in practice which choice is more convenient. Another example is the choice between $4 / \mathrm{mmm}$, the present standard choice in three dimensions, and $4 m m \perp m$, which is more in agreement with the nomenclature we propose for higher dimensions. A third example is the case of the point group of an octagonal 4D structure, which in projection gives an octagonal tiling. Its point group is generated by the transformation [8] and a twofold rotation. The transformation [8] is the same as $88^{3}$ and can be written in block form. The twofold rotation acts in both subspaces as a mirror. The point group may hence be seen either as a subdirect product $8 m\left(8^{3} \mathrm{~m}\right)$ of $8 \mathrm{~m}$ and $8 m$ or as a group generated by [8] and $m(m)$, in which case the notation becomes [8] $\mathrm{m}(\mathrm{m})$. Again, another possibility would be to indicate the generators, which act in the whole $x y z u$ space and the 2D $y z$ plane, respectively. The point group is spanned by the subgroup generated by [8] and a subgroup generated by a transformation 2. It is the smallest group containing both subgroups. This can be denoted by giving the symbols for the generating groups, separated by a dot. An alternative symbol for the group $8 m\left(8^{3} m\right)$ in this scheme is [8].2. This cannot be used for the 3D point groups: one still writes 622 (instead of 6.2) and 432 (instead of 4.3). In general, if the point group $K$ is the smallest group containing its subgroups $K_{1}$ and $K_{2}$, it is denoted by $K_{1} \cdot K_{2}$. In the particular case that $K$ is the direct product of its subgroups $K_{1}$ and $K_{2}$, it is denoted by $K_{1} \times K_{2}$. Whether [8].2 or $8 m\left(8^{3} \mathrm{~m}\right)$ or [8] $m(\mathrm{~m})$ is used is a matter of taste and the most convenient notation should again become clear in practice. Finally, it might be convenient to denote a cyclic point group by the symbol for its generator. Here, one should be careful with symbols of transformations already in use as symbols for threedimensional point groups $(222,32,422,622,432)$. The cyclic groups based on these transformations should have another notation: in standard notation, $\overline{1_{6}}, 3 \perp 2$, $4\left(\overline{1_{4}}\right), 6\left(\overline{1_{4}}\right)$ and $4(2) \perp 3$, respectively. A number of alternatives are mentioned in Table 3. Synoptic lists relating the entries in Table 3 to other notations have been compiled by several authors. In particular, Veysseyre (1998) and, separately, Wondratschek (1998) have related the entries in Table 3 to the crystal classes in Brown et al. (1978).

Irreducible point groups and point groups having invariant subspaces of dimension higher than three cannot use only the symbols of one-, two- and threedimensional point groups. Recommendations for these cases will be given in a subsequent Report.

Recommendation 5. Symbols for geometric crystal classes are symbols for representative point groups. The following symbols should be used in point groups that are the subdirect product of point groups in at most three dimensions.

(i) A point group in $n$ dimensions that can be written as the direct product of point groups acting in mutually perpendicular subspaces is called an external product of $K_{1}, K_{2}, \ldots$ and is denoted by $K_{1} \perp K_{2} \perp \ldots$ A subdirect product of $K_{1}, K_{2}, \ldots$ is a subgroup of the external product $K_{1} \perp K_{2} \perp \ldots$..

(ii) If the point group is reducible into block form with blocks of dimension three or less and not an external product of point groups, the following symbols are chosen.

For convenience, we consider the case where the point group $K$ is a subgroup of $K_{1} \perp K_{2}$. Generalization to the case with more components is straightforward. The symbol consists of a pair $H_{1}\left(H_{2}\right)$, where $H_{i}$ is the symbol for a two- or three-dimensional point group $K_{i}$ or such a symbol with digits 1 added so that $(a)$ the number of alphanumeric characters is the same in both symbols, $(b)$ the $m$ th symbol in $H_{1}$ is associated with the $m$ th symbol in $H_{2}$, and (c) the elements of $H_{i}$ that are paired with a digit 1 in the other symbol generate an invariant subgroup. 
(iii) Simplified symbols are chosen for the groups $K$ that are the direct product of a subgroup $H$ of index two and the central inversion $\overline{1_{n}}$. Such a group is denoted by $H \times \overline{1_{n}}$.

\section{Families}

The set of $n \mathrm{D}$ lattices can be partitioned into Bravais classes, lattice systems (Bravais systems in ch. 8 of ITC) and families. Lattice systems and families may likewise be characterized by the geometric crystal classes of the holohedral point groups.

Lattices for a certain holohedry have a metric tensor with a number of free parameters. This number is the dimension of the subspace of the space of second-rank symmetric tensors that transform with the identity representation. This dimension is the same for all point groups in the same geometric class. A specific choice of standard basis can show the reducibility of the space. Such a standard basis can be used for the definition of a conventional cell. For each family, a lattice may be chosen such that each other lattice of the family may be considered as related by centring. As examples, all orthorhombic lattices in three dimensions can be obtained by adding centring translations to a primitive orthorhombic lattice; the lattices of the trigonal/hexagonal family can be obtained similarly from a hexagonal lattice. The choice of standard basis is very important for the description of structures. Recommended symbols for four, five and six dimensions are given in Tables 4 to 9 , together with the metric tensors for a chosen standard basis. $6 \mathrm{D}$ families are given in Table 8 , but their metric tensors are given for a selected set only in Table 9. The others can be read off from the preceding tables. If the holohedral point group is $K_{1} \perp K_{2}$, the matrix for the metric tensor is the direct sum of the metric tensors of the lower-dimensional groups. For example, in the 6D family 6D_32 (i.e. number 32 in Table 8), the holohedral point group is $4(4) \perp 4 m$. The metric tensor for 4(4) can be found in Table 4 , and $4 m$ is a two-dimensional group. Therefore, the metric tensor for $4(4) \perp 4 m$ is

$$
\left(\begin{array}{rrrrrr}
a & 0 & f & g & 0 & 0 \\
& a & -g & f & 0 & 0 \\
& & c & 0 & 0 & 0 \\
& & & c & 0 & 0 \\
& & & & d & 0 \\
& & & & & d
\end{array}\right) .
$$

There are two types of new families in six dimensions. First, those involving $n$-fold rotations for which the Euler function $\Phi(n)$ has the value 6: the dihedral groups of order 28 and 36 . Second, the four $R$-irreducible families. The latter are the families of the hypercubic lattice (in the series 2D_4mm, 3D_m $m \overline{3} m$, 4D_23, 5D_32) with holohedral point group of order $46080=2^{6} 6$ !, the rhombohedral lattice (in the series 2D_6mm, 3D_bcc, 4D_21, 5D_31) of order $10080=2 \times 7$ !, a lattice in the series 2D_6mm, 4D_22 with holohedral point group of order $10368=3 ! \times 12^{3}$, and a symmetrized version of lattice 6D_78 with holohedral point group of order 240 (Plesken \& Hanrath, 1984), respectively.

The order in which the families are presented is preferably such that families having properties in common are grouped together. Unfortunately, there are conflicting criteria. There is no natural order. Because there is a hierarchy: family - system - geometric point group - arithmetic point group - space group, the numbering could be based on this hierarchy and in that case a numbering of the families is necessary. Simple criteria are connected to the number of free parameters in the metric tensor and to the order of the holohedral point group. The most general lattice has the maximal number of free parameters and the smallest holohedral point group, whereas a lattice becomes increasingly special if these numbers decrease and increase, respectively.

This order is related, but not identical, to that based on the reducibility of the arithmetic holohedral point group. The holohedral point group is reducible if it is equivalent to a direct sum of irreducible representations. A distinction is necessary between equivalence via rational basis transformations and that via real basis transformations. The type of reducibility is given by writing the dimension as the sum of the dimensions of the irreducible components. In three dimensions, the holohedral point group of a cubic lattice is 3-irreducible, that of an orthorhombic lattice is $1+1+1$-reducible. In four dimensions, a lattice left invariant by a point group generated by the transformation [8] is $2+2$-reducible if real transformations are considered, but if rational transformations only are allowed then it is irreducible. Further, there is a difference between rational and integer equivalence as well. In three dimensions, the orthorhombic $P$ lattice is fully $1+1+1$-reducible if integer matrices are used but the orthorhombic $F$ lattice is reducible but not fully reducible. However, this difference becomes relevant only if centrings are considered. This point will be discussed in a following Report. An alternative ordering of the families takes this reducibility as the starting point. Families with the same dimensions as the irreducible components of the holodral point groups are then grouped together. A comparison of the two schemes is given in Fig. 2 for the 4D families.

The reducibility scheme lists the dimensions of the irreducible components. This scheme can be refined as follows. Suppose that the holohedral point group $K$ can be written as an external product $K_{1} \perp K_{2}$. If one gives the reduction scheme for each of the components by putting it in parentheses, the reduction scheme gives more information. For example, in three dimensions, the holohedral point groups of the triclinic, monoclinic and 
orthorhombic families are all $1+1+1$-reducible. Addition of the new information gives the reduction scheme $(1+1+1),(1+1)+1$ or $1+1+1$, respectively.

In the opinion of the Subcommittee, both schemes for ordering the families have advantages and disadvantages but there is a preference for the scheme chosen, primarily because the number of free parameters in the metric tensor plays an important rôle. Neither of the schemes allows for a natural grouping of families in all cases. Opgenorth et al. (1998) have recently introduced, in a survey of algorithms used in the computer program $C A R A T$, an alternative grouping of crystal families in $n$ dimensions based on the decomposition pattern, groupsubgroup relations and metric tensors in their Section 2.4. The main issue in this section of the recommendations, however, is the choice of the standard basis.

A recommendation for the choice of the standard basis is given in Table 4 for four dimensions, in Table 7

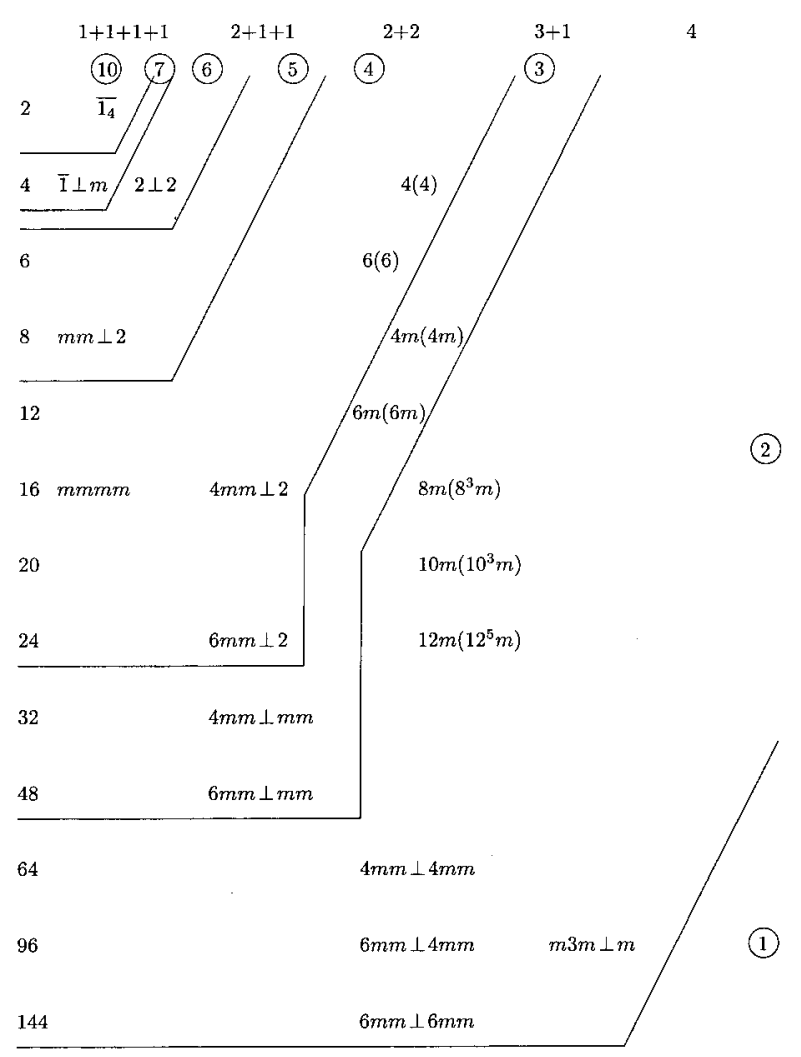

$240 \quad 4 \mathrm{D} .21$

$288-4 \mathrm{D} \_22$

$1152 \quad 4$ D_23

Fig. 2. The 23 four-dimensional families, in rows according to the order of the holohedral point group, and in columns according to the $R$-reducibility. Lines separate families with different numbers of free parameters (indicated in the circles). for five dimensions, and in Table 9 for six dimensions. In general, the chosen standard basis for a family is that of a lattice with the highest symmetry. Sometimes this is not the most convenient choice. In the 4D family No. 23, there are two lattice systems, one is the hypercubic system with holohedry of order 384, the other one with holohedry of order 1152. The transformations of the groups of the first system have a very simple form when described on a basis of the hypercubic lattice. In each row and column, there is exactly one non-zero element. If one chooses a basis in the other system this is not the case. However, point groups that do not leave the hypercubic lattice invariant are represented by noninteger matrices if one uses the hypercubic basis. Therefore, we propose using the hypercubic basis for all point groups belonging to point-group system 23_1, and the second basis (that of the lattice with holohedral point group of order 1152) for the point groups in point group system 23_2 only. The alternatives are given in Table 5.

The full list of 4D families was given for the first time by Brown et al. (1978). Comparison of the numbering in that publication with the numbering in Table 4 is given in Table 6.

Recommendation 6. Recommended symbols for families are the same as those for corresponding holohedral point groups.

Recommendation 7. The family order is first according to the number of free parameters in the metric tensor (in decreasing order), and second according to the order of the holohedral point group (in increasing order).

Recommendation 8. The recommended standard basis for each family is chosen such that the reducibility becomes maximally evident. Orthogonal invariant subspaces are ordered according to decreasing orders of the corresponding point groups. The conventional cell for a lattice system is the cell of the corresponding family. As an alternative, a special basis for each lattice system in the family may be used. The recommended bases and metric tensors for four-, five- and six-dimensional families are given in Tables 4, 7 and 9.

\section{Relation with symmetries of aperiodic crystals}

$n \mathrm{D}$ crystallography as such has become a topic for research. On the other hand, $n \mathrm{D}$ space groups are widely used for the description of aperiodic crystals. In this section, we discuss the relation between the general problem and the applications to aperiodic structures. It should be noted that the use of $n \mathrm{D}$ crystallographic groups is not the only method for describing aperiodic structures. Colour symmetry or Fourier transforms in 3D space are also used. However, these are strongly related to a description in terms of higher-dimensional crystallography. Here we only note one of the applications of 
Table 4. Standard metric tensors for the 23 four-dimensional families

\begin{tabular}{|c|c|c|c|c|c|c|}
\hline \multirow[b]{2}{*}{ No. } & \multirow[b]{2}{*}{$N_{f p} \dagger$} & \multirow[b]{2}{*}{$|H| \ddagger$} & \multirow{2}{*}{$\begin{array}{l}\text { Holohedral } \\
\text { point group }\end{array}$} & \multirow[b]{2}{*}{ Metric tensor } & \multicolumn{2}{|c|}{ Decomposition } \\
\hline & & & & & $\overline{\text { Real }}$ & Rational \\
\hline 1 & 10 & 2 & $\overline{1_{4}}$ & $\left(\begin{array}{llll}a & e & f & g \\
& b & h & i \\
& & c & j \\
& & & d\end{array}\right)$ & $(1+1+1+1)$ & $(1+1+1+1)$ \\
\hline 2 & 7 & 4 & $\overline{1} \perp m$ & $\left(\begin{array}{llll}a & e & f & 0 \\
& b & h & 0 \\
& & c & 0 \\
& & & d\end{array}\right)$ & $(1+1+1)+1$ & $(1+1+1)+1$ \\
\hline 3 & 6 & 4 & $2 \perp 2$ & $\left(\begin{array}{llll}a & e & 0 & 0 \\
& b & 0 & 0 \\
& & c & j \\
& & & d\end{array}\right)$ & $(1+1)+(1+1)$ & $(1+1)+(1+1)$ \\
\hline 4 & 5 & 8 & $m m \perp 2$ & $\left(\begin{array}{llll}a & 0 & 0 & 0 \\
& b & 0 & 0 \\
& & c & j \\
& & & d\end{array}\right)$ & $1+1+(1+1)$ & $1+1+(1+1)$ \\
\hline 5 & 4 & 4 & $4(4)$ & $\left(\begin{array}{cccc}a & 0 & f & g \\
& a & -g & f \\
& & c & 0 \\
& & & c\end{array}\right)$ & $(2+2)$ & $(2+2)$ \\
\hline 6 & 4 & 6 & $6(6)$ & $\left(\begin{array}{ccc}a & -\frac{1}{2} a & f \\
& a & -f- \\
& & c\end{array}\right.$ & $(2+2)$ & $(2+2)$ \\
\hline 7 & 4 & 16 & $\mathrm{mmmm}$ & $\left(\begin{array}{llll}a & 0 & 0 & 0 \\
& b & 0 & 0 \\
& & c & 0 \\
& & & d\end{array}\right)$ & $1+1+1+1$ & $1+1+1+1$ \\
\hline 8 & 4 & 16 & $4 m m \perp 2$ & $\left(\begin{array}{cccc}a & 0 & 0 & 0 \\
& a & 0 & 0 \\
& & c & j \\
& & & d\end{array}\right)$ & $2+(1+1)$ & $2+(1+1)$ \\
\hline 9 & 4 & 24 & $6 m m \perp 2$ & $\left(\begin{array}{cccc}a & -\frac{1}{2} a & 0 & 0 \\
& a & 0 & 0 \\
& & c & j \\
& & & d\end{array}\right.$ & $2+(1+1)$ & $2+(1+1)$ \\
\hline 10 & 3 & 8 & $4 m(4 m)$ & $\left(\begin{array}{llll}a & 0 & f & 0 \\
& a & 0 & f \\
& & c & 0 \\
& & & c\end{array}\right)$ & $(2+2)$ & $(2+2)$ \\
\hline 11 & 3 & 12 & $6 m(6 m)$ & $\begin{array}{cc}a & -\frac{1}{2} f \\
& c\end{array}$ & $(2+2)$ & $(2+2)$ \\
\hline 12 & 3 & 32 & $4 m m \perp m m$ & $\left(\begin{array}{cccc}a & 0 & 0 & 0 \\
& a & 0 & 0 \\
& & c & 0 \\
& & & d\end{array}\right)$ & $2+1+1$ & $2+1+1$ \\
\hline 13 & 3 & 48 & $6 m m \perp m m$ & $\left(\begin{array}{cccc}a & -\frac{1}{2} a & 0 & 0 \\
& a & 0 & 0 \\
& & c & 0 \\
& & & d\end{array}\right.$ & $2+1+1$ & $2+1+1$ \\
\hline 14 & 2 & 16 & $8 m\left(8^{3} m\right)$ & $\left(\begin{array}{cccc}a & e & 0 & -e \\
& a & e & 0 \\
& & a & e \\
& & & a\end{array}\right)$ & $(2+2)$ & 4 \\
\hline
\end{tabular}


Table 4 (cont.)

\begin{tabular}{|c|c|c|c|c|c|c|}
\hline \multirow[b]{2}{*}{ No. } & \multirow[b]{2}{*}{$N_{f p} \dagger$} & \multirow[b]{2}{*}{$|H| \ddagger$} & \multirow{2}{*}{$\begin{array}{l}\text { Holohedral } \\
\text { point group }\end{array}$} & \multirow[b]{2}{*}{ Metric tensor } & \multicolumn{2}{|c|}{ Decomposition } \\
\hline & & & & & Real & Rational \\
\hline 15 & 2 & 20 & $10 m\left(10^{3} m\right)$ & $\left(\begin{array}{lll}a & e & f \\
& a & e \\
& & a\end{array}\right) \quad 2(e+f)+a=0$ & $(2+2)$ & 4 \\
\hline 16 & 2 & 24 & $12 m\left(12^{5} m\right)$ & $\left(\begin{array}{cccc}a & e & \frac{1}{2} a & 0 \\
& a & e & \frac{1}{2} a \\
& & a & e \\
& & & a\end{array}\right)$ & $(2+2)$ & 4 \\
\hline 17 & 2 & 64 & $4 m m \perp 4 m m$ & $\left(\begin{array}{cccc}a & 0 & 0 & 0 \\
& a & 0 & 0 \\
& & c & 0 \\
& & & c\end{array}\right)$ & $2+2$ & $2+2$ \\
\hline 18 & 2 & 96 & $6 \mathrm{~mm} \perp 4 \mathrm{~mm}$ & $\left(\begin{array}{cccc}a & -\frac{1}{2} a & 0 & 0 \\
& a & 0 & 0 \\
& & c & 0 \\
& & & c\end{array}\right)$ & $2+2$ & $2+2$ \\
\hline 19 & 2 & 96 & $m \overline{3} m \perp m$ & $\left(\begin{array}{llll}a & 0 & 0 & 0 \\
& a & 0 & 0 \\
& & a & 0 \\
& & & d\end{array}\right)$ & $3+1$ & $3+1$ \\
\hline 20 & 2 & 144 & $6 m m \perp 6 m m$ & $\left(\begin{array}{cccc}a & -\frac{1}{2} a & 0 & 0 \\
& a & 0 & 0 \\
& & c & -\frac{1}{2} c \\
& & & c\end{array}\right)$ & $2+2$ & $2+2$ \\
\hline 21 & 1 & 240 & - & $\begin{array}{ccc}a & \frac{1}{2} a & \frac{1}{2} a \\
& a & \frac{1}{2} a\end{array}$ & 4 & 4 \\
\hline 22 & 1 & 288 & - & $\left(\begin{array}{cccc}a & -\frac{1}{2} a & 0 & 0 \\
& a & 0 & 0 \\
& & a & -\frac{1}{2} a \\
& & & a\end{array}\right)$ & 4 & 4 \\
\hline 23 & 1 & $1152 \S$ & - & $\left(\begin{array}{cccc}a & 0 & 0 & 0 \\
& a & 0 & 0 \\
& & a & 0 \\
& & & a\end{array}\right)$ & 4 & 4 \\
\hline
\end{tabular}

$\dagger N_{f p}=$ number of free parameters. $\$|H|=$ order of the maximal holohedral point group. $\quad \S$ The order of the holohedral point group of the lattice spanned by the standard basis is 384 .

the groups discussed in this Report. We do not specifically recommend this method, but simply draw attention to the fact that the symbols used by many crystallographers for quasiperiodic systems are closely related to those that arise naturally for $n \mathrm{D}$ groups with a reducible point group.

A lattice-periodic function in $n$ variables gives an aperiodic structure if the space is intersected by an oblique subspace. Consider a function $f\left(x_{1}, x_{2}\right)$ of two variables which is periodic in its two arguments: $f\left(x_{1}+1, x_{2}\right)=f\left(x_{1}, x_{2}+1\right)=f\left(x_{1}, x_{2}\right)$. Take two real numbers $\alpha_{1}$ and $\alpha_{2}$ such that $\alpha_{1} / \alpha_{2}$ is irrational and define a function $g(x)$ of one variable through $g(x):=f\left(\alpha_{1} x, \alpha_{2} x\right)$. Its Fourier series follows from that of $f\left(x_{1}, x_{2}\right)$ :

$$
\begin{array}{r}
f\left(x_{1}, x_{2}\right)=\sum_{h_{1}, h_{2}} c\left(h_{1}, h_{2}\right) \exp \left[2 \pi i\left(h_{1} x_{1}+h_{2} x_{2}\right)\right] \\
\rightarrow g(x)=\sum_{h_{1}, h_{2}} c\left(h_{1}, h_{2}\right) \exp \left[2 \pi i\left(h_{1} \alpha_{1}+h_{2} \alpha_{2}\right) x\right], \\
\text { integer } h_{i} .
\end{array}
$$

This expression is a special case of a quasiperiodic function

$$
\rho(\vec{r})=\sum_{\vec{k} \in M^{*}} \hat{\rho}(\vec{k}) \exp [i \vec{k} \cdot \vec{r}],
$$

where the set $M^{*}$ is defined as

$$
M^{*}=\left\{\vec{k} \mid \vec{k}=\sum_{i=1}^{n} h_{i} \vec{a}_{i}^{*}\right\} .
$$


Table 5. Alternatives for four-dimensional family 23

Order

384

1152
Generators holohedral point group

$$
\begin{aligned}
& \left(\begin{array}{rrrr}
0 & 1 & 0 & 0 \\
1 & 0 & 0 & 0 \\
0 & 0 & 1 & 0 \\
0 & 0 & 0 & 1
\end{array}\right) \\
& \left(\begin{array}{rrrr}
-1 & 0 & 0 & 0 \\
0 & 1 & 0 & 0 \\
0 & 0 & 1 & 0 \\
0 & 0 & 0 & 1
\end{array}\right) \\
& \left(\begin{array}{rrrr}
1 & 0 & 0 & 0 \\
0 & 0 & 0 & -1 \\
0 & 1 & 1 & 1 \\
0 & -1 & 0 & 0
\end{array}\right) \\
& \left(\begin{array}{rrrr}
r & 0 & 0 & 1 \\
1 & 1 & 0 & -1 \\
-1 & 0 & 1 & 1 \\
1 & 0 & 0 & 0
\end{array}\right)
\end{aligned}
$$

$\left(\begin{array}{llll}0 & 0 & 0 & 1 \\ 1 & 0 & 0 & 0 \\ 0 & 1 & 0 & 0 \\ 0 & 0 & 1 & 0\end{array}\right)$

$\left(\begin{array}{rrrr}0 & 0 & 1 & 0 \\ 1 & 0 & -1 & -1 \\ 0 & 1 & 1 & 1 \\ 0 & 0 & 0 & -1\end{array}\right)$
Metric tensor

$$
\left(\begin{array}{llll}
a & 0 & 0 & 0 \\
& a & 0 & 0 \\
& & a & 0 \\
& & & a
\end{array}\right)
$$$$
\left(\begin{array}{cccc}
a & \frac{1}{2} a & 0 & -\frac{1}{2} a \\
& a & \frac{1}{2} a & 0 \\
& & a & \frac{1}{2} a \\
& & & a
\end{array}\right)
$$

The generators of the group of order 1152 are not all integral on the first lattice basis.

Table 6. Correspondence between the numbers of the families in Table 4 and those in Brown et al. (1978): BBNWZ

$\begin{array}{lllllllll}\text { Table } 4 & 1 & 2 & 3 & 4 & 5 & 6 & 7 & 8 \\ \text { BBNWZ } & \text { I } & \text { II } & \text { III } & \text { IV } & \text { VIII } & \text { IX } & \text { V } & \text { VI } \\ \text { Table } 4 & 9 & 10 & 11 & 12 & 13 & 14 & 15 & 16 \\ \text { BBNWZ } & \text { VII } & \text { XII } & \text { XIII } & \text { X } & \text { XI } & \text { XVIII } & \text { XIX } & \text { XX } \\ \text { Table } 4 & 17 & 18 & 19 & 20 & 21 & 22 & 23 & \\ \text { BBWNZ } & \text { XIV } & \text { XV } & \text { XVII } & \text { XVI } & \text { XXII } & \text { XXI } & \text { XXIII }\end{array}$

If $n$ is larger than the dimension of the space, the function is not lattice periodic but is quasiperiodic. The set $M^{*}$ is not a reciprocal lattice, but is the projection of an $n \mathrm{D}$ reciprocal lattice $\Sigma^{*}$. A vector $\vec{k}$ in $M^{*}$ can be written as the projection $\vec{k}=\pi \vec{k}_{s}$ and the $n \mathrm{D}$ reciprocal-lattice vector $\vec{k}_{s}$ is a linear combination of basis vectors: $\vec{k}_{s}=\sum h_{i} \vec{a}_{i s}^{*}$. On the other hand, if the diffraction pattern has intensities that are the square of the modulus of a function $\hat{\rho}(\vec{k})$ on a set $M^{*}$, then there is an $n \mathrm{D}$ periodic function $\rho\left(\vec{r}_{s}\right)$ that corresponds to the $n \mathrm{D}$ Fourier transform:

$$
\rho\left(\vec{r}_{s}\right):=\sum_{\vec{k}_{s} \in \Sigma^{*}} \hat{\rho}\left(\pi \vec{k}_{s}\right) \exp \left[i \vec{k}_{s} \cdot \vec{r}_{s}\right]
$$

This function has lattice periodicity in $n$ dimensions and the lattice $\Sigma$ has $\Sigma^{*}$ as its reciprocal lattice. Moreover, as can be seen from the definition, the restriction of the $n \mathrm{D}$ function to the hyperplane on which the reciprocal lattice is projected is just the starting function $\rho(\vec{r})$. Consequently, a quasiperiodic function is the restriction of an $n \mathrm{D}$ lattice periodic function to physical space, and the number of dimensions is equal to the number of indices $h_{i}$ necessary for indexing the diffraction pattern.

Because there is a one-to-one correspondence between the function in physical space and the function in higher-dimensional space, which is essentially given by its value in the unit cell, the symmetry of the quasiperiodic structure can be identified with that of the higher-dimensional structure. This symmetry group is an $n \mathrm{D}$ space group, with an associated $n \mathrm{D}$ point group. Because the construction is based on a projection from higher-dimensional space, it is intuitively clear that symmetry operators cannot mix the physical space and the space of the additional dimensions. This statement can be made rigorous. It implies that the point groups are necessarily $R$-reducible. A point group then consists of pairs $\left(R, R_{\perp}\right)$ of an orthogonal transformation in physical space and another in the additional space. The elements $R$ form a finite point group $K_{E}$ and the elements $R_{\perp}$ a finite point group $K_{\perp}$. Therefore, the point groups for aperiodic structures are subdirect products of $K_{E}$ and $K_{\perp}$.

The literature contains hundreds of examples of such aperiodic crystals. Two follow: 
Table 7. Families in five dimensions

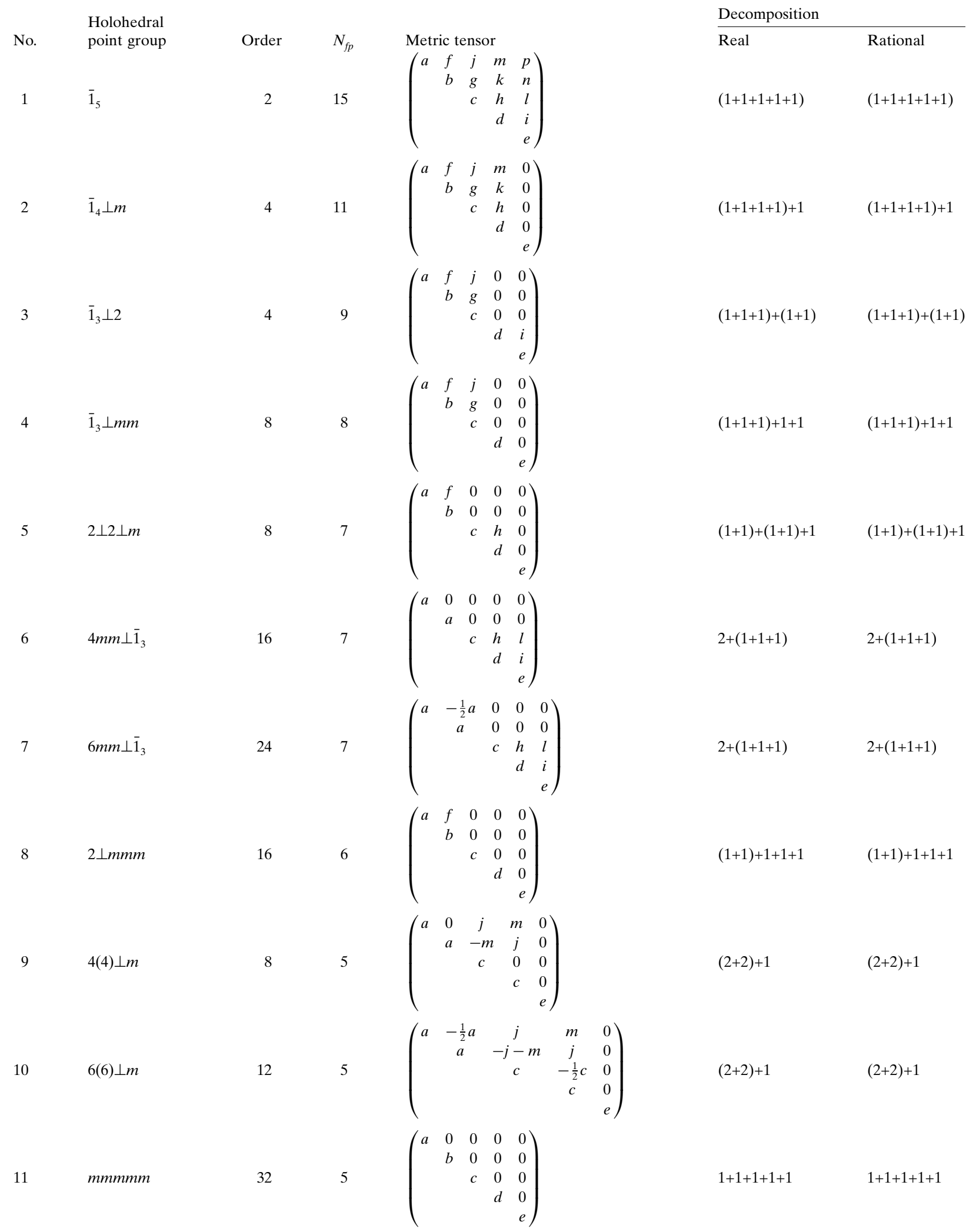


Table 7 (cont.)

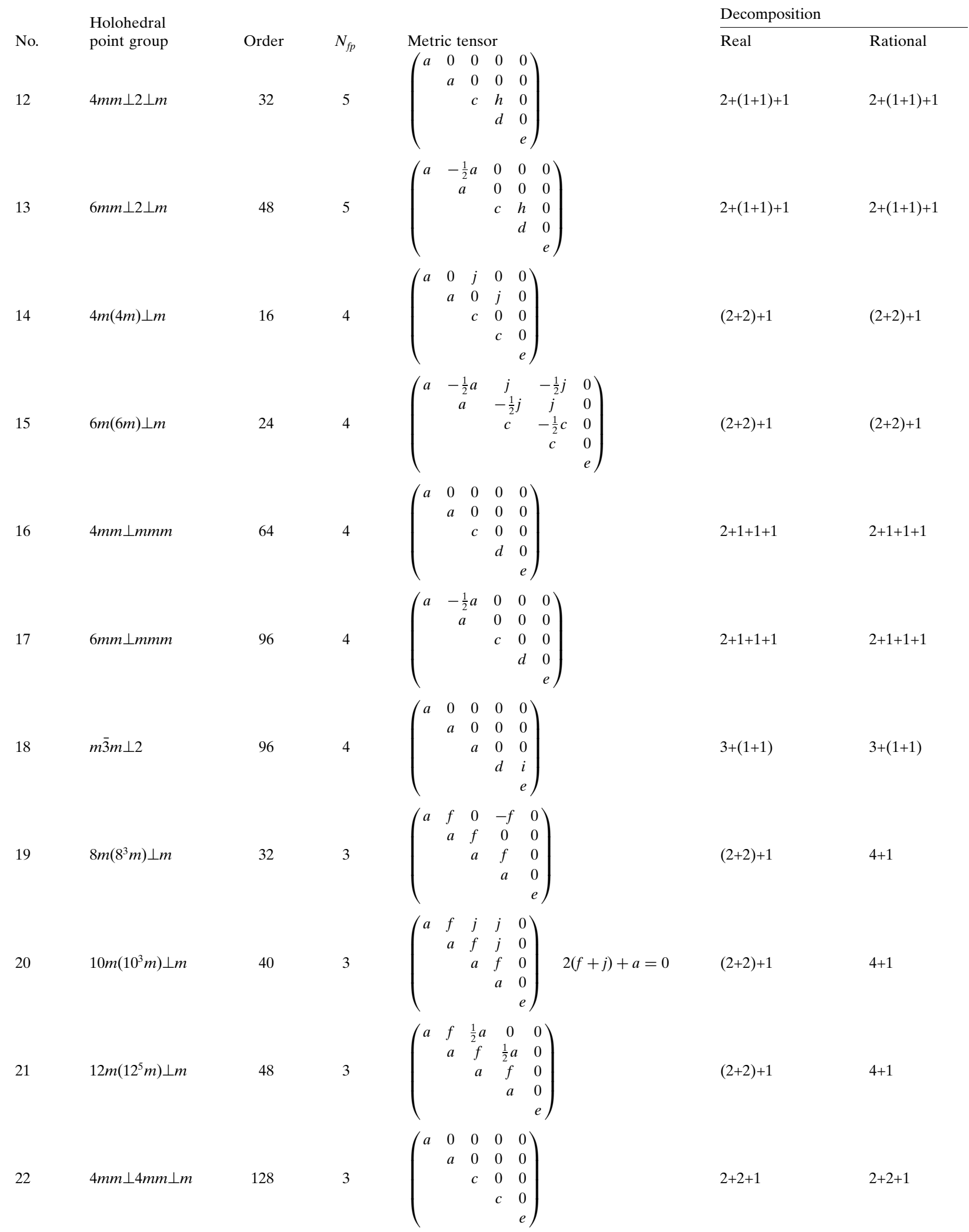


Table 7 (cont.)

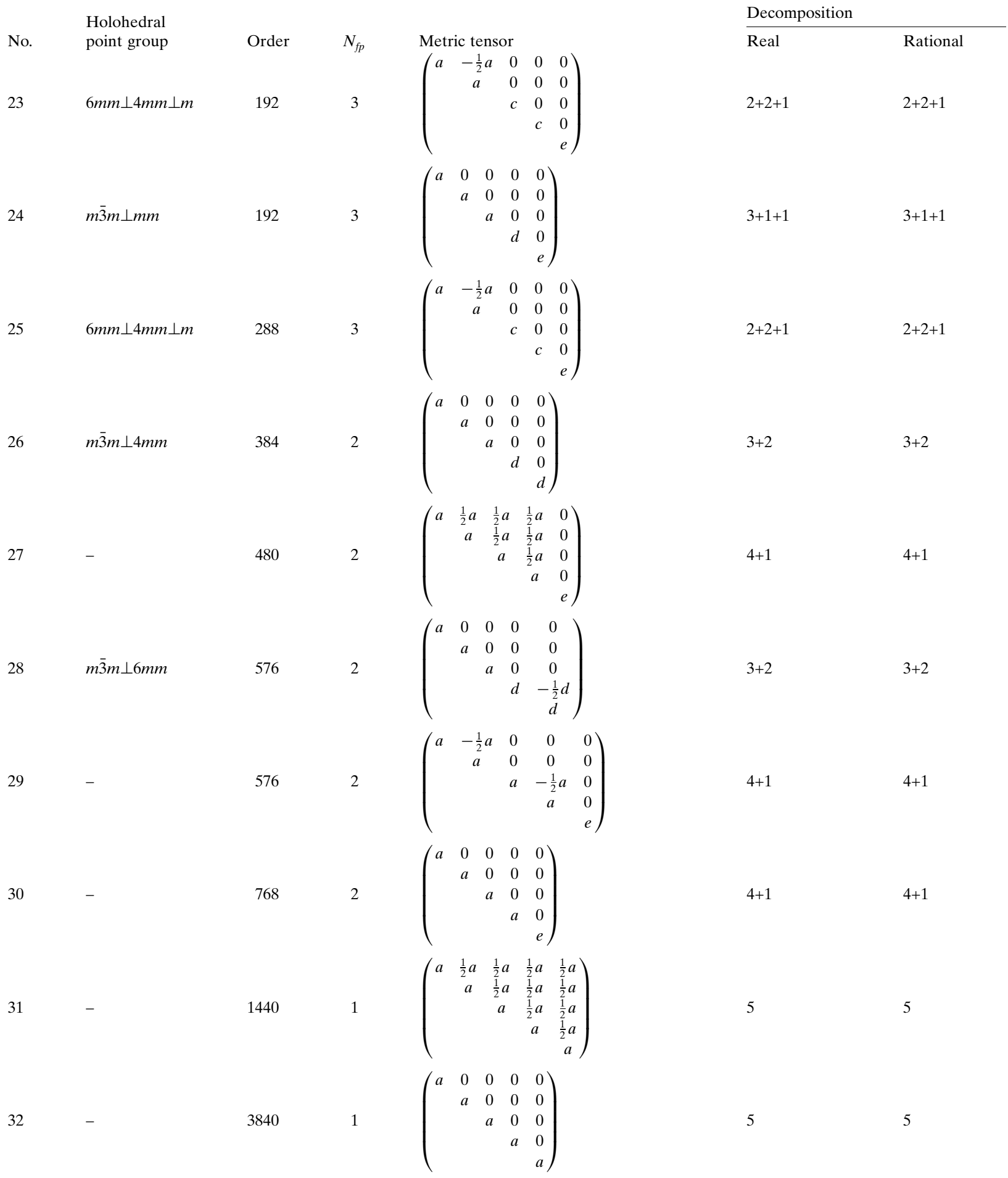

(i) $\mathrm{K}_{2} \mathrm{SeO}_{4}$ has a modulated phase in a certain temperature region. The structure in this phase can be described as derived from a basic structure with space group Pcmn via a periodic displacement modulation with wavevector $\gamma \vec{c}^{*}$. The diffraction pattern needs four indices, because a diffraction vector can be written as 
Table 8. Families in six dimensions

\begin{tabular}{|c|c|c|c|c|}
\hline No. & $N_{f p}$ & $|H|$ & $R$-reducible & Symbol \\
\hline 1 & 21 & 2 & $(1+1+1+1+1+1)$ & $\overline{1_{6}}$ \\
\hline 2 & 16 & 4 & $(1+1+1+1+1)+1$ & $\overline{1_{5}} \perp m$ \\
\hline 3 & 13 & 4 & $(1+1+1+1)+(1+1)$ & $\overline{1_{4}} \perp 2$ \\
\hline 4 & 12 & 4 & $(1+1+1)+(1+1+1)$ & $\overline{1_{3}} \perp \overline{1_{3}}$ \\
\hline 5 & 12 & 8 & $(1+1+1+1)+1+1$ & $\overline{1_{4}} \perp m m$ \\
\hline 6 & 11 & 16 & $2+(1+1+1+1)$ & $4 m \perp \overline{1_{4}}$ \\
\hline 7 & 11 & 24 & $2+(1+1+1+1)$ & $6 m \perp \overline{1_{4}}$ \\
\hline 8 & 10 & 8 & $(1+1+1)+(1+1)+1$ & $\overline{1_{3}} \perp 2 \perp m$ \\
\hline 9 & 9 & 4 & $(2+2+2)$ & $4(4)(4)$ \\
\hline 10 & 9 & 6 & $(2+2+2)$ & $6(6)(6)$ \\
\hline 11 & 9 & 8 & $(1+1)+(1+1)+(1+1)$ & $2 \perp 2 \perp 2$ \\
\hline 12 & 9 & 16 & $(1+1+1)+1+1+1$ & $\overline{1_{3}} \perp \mathrm{mmm}$ \\
\hline 13 & 8 & 16 & $(1+1)+(1+1)+1+1$ & $2 \perp 2 \perp m m$ \\
\hline 14 & 8 & 32 & $2+(1+1+1)+1$ & $4 m \perp \overline{1_{3}} \perp m$ \\
\hline 15 & 8 & 48 & $2+(1+1+1)+1$ & $6 m \perp \overline{1_{3}} \perp m$ \\
\hline 16 & 7 & 8 & $(2+2)+(1+1)$ & $4(4) \perp 2$ \\
\hline 17 & 7 & 12 & $(2+2)+(1+1)$ & $6(6) \perp 2$ \\
\hline 18 & 7 & 32 & $(1+1)+1+1+1+1$ & $2 \perp m m m m$ \\
\hline 19 & 7 & 32 & $2+(1+1)+(1+1)$ & $4 m \perp 2 \perp 2$ \\
\hline 20 & 7 & 48 & $2+(1+1)+(1+1)$ & $6 m \perp 2 \perp 2$ \\
\hline 21 & 7 & 96 & $3+(1+1+1)$ & $m 3 m \perp 1_{3}$ \\
\hline 22 & 6 & 8 & $(2+2+2)$ & $4 m(4 m)(4 m)$ \\
\hline 23 & 6 & 12 & $(2+2+2)$ & $6 m(6 m)(6 m)$ \\
\hline 24 & 6 & 16 & $(2+2)+1+1$ & $4(4) \perp m m$ \\
\hline 25 & 6 & 16 & $(2+2)+(1+1)$ & $4 m(4 m) \perp 2$ \\
\hline 26 & 6 & 24 & $(2+2)+1+1$ & 6(6) $\perp m m$ \\
\hline 27 & 6 & 24 & $(2+2)+(1+1)$ & $6 m(6 m) \perp 2$ \\
\hline 28 & 6 & 64 & $1+1+1+1+1+1$ & тттттт \\
\hline 29 & 6 & 64 & $2+(1+1)+1+1$ & $4 m \perp 2 \perp m m$ \\
\hline 30 & 6 & 96 & $2+(1+1)+1+1$ & $6 m \perp 2 \perp m m$ \\
\hline 31 & 5 & 32 & $(2+2)+1+1$ & $4 m(4 m) \perp m m$ \\
\hline 32 & 5 & 32 & $(2+2)+2$ & $4(4) \perp 4 m$ \\
\hline 33 & 5 & 32 & {$[2+2]+(1+1)$} & $8 m\left(8^{3} m\right) \perp 2$ \\
\hline 34 & 5 & 40 & {$[2+2]+(1+1)$} & $10 m\left(10^{3} m\right) \perp 2$ \\
\hline 35 & 5 & 48 & $(2+2)+1+1$ & $6 m(6 m) \perp m m$ \\
\hline 36 & 5 & 48 & $(2+2)+2$ & $4(4) \perp 6 m$ \\
\hline 37 & 5 & 48 & $(2+2)+2$ & $6(6) \perp 4 m$ \\
\hline 38 & 5 & 48 & {$[2+2]+(1+1)$} & $12 m\left(12^{5} m\right) \perp 2$ \\
\hline 39 & 5 & 72 & $(2+2)+2$ & $6(6) \perp 6 m$ \\
\hline 40 & 5 & 128 & $2+1+1+1+1$ & $4 m \perp m m m m$ \\
\hline 41 & 5 & 128 & $2+2+(1+1)$ & $4 m \perp 4 m \perp 2$ \\
\hline 42 & 5 & 192 & $2+1+1+1+1$ & $6 m \perp m m m m$ \\
\hline 43 & 5 & 192 & $2+2+(1+1)$ & $6 m \perp 4 m \perp 2$ \\
\hline 44 & 5 & 192 & $3+(1+1)+1$ & $m 3 m \perp 2 \perp m$ \\
\hline 45 & 5 & 288 & $2+2+(1+1)$ & $6 m \perp 6 m \perp 2$ \\
\hline 46 & 4 & 64 & $(2+2)+2$ & $4 m(4 m) \perp 4 m$ \\
\hline 47 & 4 & 64 & {$[2+2]+1+1$} & $8 m\left(8^{3} m\right) \perp m m$ \\
\hline 48 & 4 & 80 & {$[2+2]+1+1$} & $10 m\left(10^{3} m\right) \perp m m$ \\
\hline 49 & 4 & 96 & {$[2+2]+1+1$} & $12 m\left(12^{5} m\right) \perp m m$ \\
\hline 50 & 4 & 96 & $(2+2)+2$ & $4 m(4 m) \perp 6 m$ \\
\hline 51 & 4 & 96 & $(2+2)+2$ & $6 m(6 m) \perp 4 m$ \\
\hline 52 & 4 & 144 & $(2+2)+2$ & $6 m(6 m) \perp 6 m$ \\
\hline 53 & 4 & 256 & $2+2+1+1$ & $4 m \perp 4 m \perp m m$ \\
\hline 54 & 4 & 384 & $2+2+1+1$ & $6 m \perp 4 m \perp m m$ \\
\hline 55 & 4 & 384 & $3+1+1+1$ & $m 3 m \perp m m m$ \\
\hline 56 & 4 & 480 & $4+(1+1)$ & {$\left[4 \mathrm{D} \_21\right] \perp 2$} \\
\hline 57 & 4 & 576 & $2+2+1+1$ & $6 m \perp 6 m \perp m m$ \\
\hline 58 & 4 & 576 & $4+(1+1)$ & {$\left[4 \mathrm{D} \_22\right] \perp 2$} \\
\hline 59 & 4 & 768 & $4+(1+1)$ & {$\left[4 \mathrm{D} \_23\right] \perp 2$} \\
\hline 60 & 3 & 28 & {$[2+2+2]$} & $14 m(14 m)(14 m)$ \\
\hline 61 & 3 & 36 & {$[2+2+2]$} & $18 m(18 m)(18 m)$ \\
\hline 62 & 3 & 48 & $(3+3)$ & $m \overline{3} m(m \overline{3} m)$ \\
\hline 63 & 3 & 128 & {$[2+2]+2$} & $8 m\left(8^{3} m\right) \perp 4 m$ \\
\hline 64 & 3 & 160 & {$[2+2]+2$} & $10 m\left(10^{3} m\right) \perp 4 m$ \\
\hline 65 & 3 & 192 & {$[2+2]+2$} & $12 m\left(12^{5} m\right) \perp 4 m$ \\
\hline 66 & 3 & 192 & {$[2+2]+2$} & $8 m\left(8^{3} m\right) \perp 6 m$ \\
\hline
\end{tabular}


Table 8 (cont.)

$\begin{array}{llrl}\text { No. } & N_{f p} & |H| & R \text {-reducible } \\ 67 & 3 & 240 & {[2+2]+2} \\ 68 & 3 & 288 & {[2+2]+2} \\ 69 & 3 & 512 & 2+2+2 \\ 70 & 3 & 768 & 2+2+2 \\ 71 & 3 & 768 & 3+2+1 \\ 72 & 3 & 960 & 4+1+1 \\ 73 & 3 & 1152 & 2+2+2 \\ 74 & 3 & 1152 & 3+2+1 \\ 75 & 3 & 1152 & 4+1+1 \\ 76 & 3 & 1536 & 4+1+1 \\ 77 & 3 & 1728 & 2+2+2 \\ 78 & 2 & 120 & {[3+3]} \\ 79 & 2 & 1920 & 4+2 \\ 80 & 2 & 2304 & 3+3 \\ 81 & 2 & 2304 & 4+2 \\ 82 & 2 & 2880 & 4+2 \\ 83 & 2 & 2880 & 5+1 \\ 84 & 2 & 3072 & 4+2 \\ 85 & 2 & 3456 & 4+2 \\ 86 & 2 & 4608 & 4+2 \\ 87 & 2 & 7680 & 5+1 \\ 88 & 1 & 240 & 6 \\ 89 & 1 & 10080 & 6 \\ 90 & 1 & 10368 & 6 \\ 91 & 1 & 46080 & 6\end{array}$

Symbol
$10 m\left(10^{3} m\right) \perp 6 m$
$12 m\left(12^{5} m\right) \perp 6 m$
$4 m \perp 4 m \perp 4 m$
$6 m \perp 4 m \perp 4 m$
$m \overline{3} m \perp 4 m \perp m$
$\left[4 \mathrm{D} \_21\right] \perp m m$
$6 m \perp 6 m \perp 4 m$
$m \overline{3} m \perp 6 m \perp m$
$\left[4 \mathrm{D} \_22\right] \perp m m$
$[4 \mathrm{D} 23] \perp m m$
$6 m \perp 6 m \perp 6 m$
$5 \overline{3} m\left(5^{2} \overline{3} m\right)$
$[4 \mathrm{D} 221] \perp 4 m$
$m \overline{3} m \perp m \overline{3} m$
$\left[4 \mathrm{D} \_22\right] \perp 4 m$
$\left[4 \mathrm{D} \_21\right] \perp 6 m$
[5D_31] $\perp m$
$\left[4 \mathrm{D} \_23\right] \perp 4 m$
$[4 \mathrm{D} 22] \perp 6 m$
$\left[4 \mathrm{D} \_23\right] \perp 6 m$
$\left[5 \mathrm{D} \_32\right] \perp m$
[6D_88]
[6D_89]
[6D_90]
[6D_91]

Symbol

$12 m\left(12^{5} m\right) \perp 6 m$

$4 m \perp 4 m \perp 4 m$

$m \overline{3} m \perp 4 m \perp m$

[4D_21] $\perp m m$

[4D 22] $\perp m m$

[4D_23] $\perp m m$

[4D 21$] \perp 4 m$

$m 3 m \perp m 3 m$

[4D 22] $\perp 4 m$

[5D 31$]+m$

[4D_23] $\perp 4 m$

[4D 22$] \perp 6 m$

$\left[4 \mathrm{D} \_23\right] \perp 6 m$

[5D_32]د

[6D_89]

[6D_91]

$\mathbb{Z}$-reducibility is indicated by [ ]: [2+2] is $(2+2)$ - $R$-reducible, but $4 \mathrm{D} \mathbb{Z}$-irreducible. Symbols for $R$-reducible groups have not yet been settled for $n>3$ and are indicated as [ $n \mathrm{D} \_$No.].

$$
\vec{k}=h \vec{a}^{*}+k \vec{b}^{*}+l \vec{c}^{*}+m\left(\gamma \vec{c}^{*}\right) .
$$

The lattice periodic function in four dimensions has a point group generated by the pairs $\left(m_{x}, 1\right),\left(m_{y}, 1\right)$ and $\left(m_{z},-1\right)$. Such a point group is the subdirect product of the $3 \mathrm{D}$ point group $\mathrm{mmm}$ and the $1 \mathrm{D}$ point group $m$. In the recommended notation, it is the group $m m \perp 2$. In the literature for modulated structures, it usually has the symbol $m m m(11 \overline{1})$.

(ii) The quasicrystalline phase of AlMnPd has a diffraction pattern with icosahedral symmetry, requiring six indices. The number of dimensions for the space group is hence $n=6$. The point group is the subdirect product of the icosahedral point groups $5 \overline{3} \mathrm{~m}$ in the physical and the $3 \mathrm{D}$ additional space. It is generated by pairs $5\left(5^{2}\right), \overline{3}(\overline{3})$ and $m(m)$. Therefore, the recommended notation for the point group is $5 \overline{3} m\left(5^{2} \overline{3} \mathrm{~m}\right)$. In the literature, it is generally denoted by $5 \overline{3} \mathrm{~m}$, which is shorter but is also ambiguous because it is the symbol for a 3D point group and it suppresses important information as the components in additional space are not indicated. The nomenclature for aperiodic systems can therefore benefit from the more general approach discussed in this Report in terms of $n \mathrm{D}$ crystallography. On the other hand, the notation schemes are not so different that it is difficult to change from one to the other.

\section{Conclusions and recommendations}

Recommendations for the symbols of $n$-dimensional orthogonal transformations, $n$-dimensional geometric crystal classes, the order of $n$-dimensional families, and the standard bases in four, five and six dimensions are given at the end of $\S \S 2,3$ and 4. Specific recommendations for dimensions up to six are given in the tables.

The recommendations are summarized as follows.

(I) An orthogonal transformation of finite order that can be written after a suitable real basis transformation as the direct sum

$$
\begin{array}{r}
S=\bigoplus_{i=1}^{r}\left(\begin{array}{cc}
\cos 2 \pi p_{i} / q_{i} & -\sin 2 \pi p_{i} / q_{i} \\
\sin 2 \pi p_{i} / q_{i} & \cos 2 \pi p_{i} / q_{i}
\end{array}\right) \oplus \bigoplus_{j=1}^{m} a_{j} \oplus \bigoplus_{k=1}^{l} b_{k}, \\
a_{j}=-1, b_{k}=1,2 r+m+l=n, \quad \text { (20) }
\end{array}
$$

should be written in full as

$$
q_{1}^{p_{1}} q_{2}^{p_{2}} 22 \ldots
$$

with $m / 22$ 's when $m$ is even,

$$
\overline{d_{1}^{c_{1}} d_{2}^{c_{2}} \ldots 22 \ldots}
$$

with $(m-1) / 2$ 2's when $m$ is odd, and with $c_{i} / d_{i}=p_{i} / q_{i}+\frac{1}{2}(\bmod 1)$, but it is

$$
1_{n}
$$


Table 9. Metrical tensors for some six-dimensional families

No.

9

Standard tensor

$\left(\begin{array}{cccccc}a & 0 & d & -g & e & h \\ & a & g & d & -h & e \\ & & b & 0 & f & i \\ & & & b & -i & f \\ & & & & c & 0 \\ & & & & & c\end{array}\right)$

$22 \quad\left(\begin{array}{llllll}a & 0 & d & 0 & e & 0 \\ & a & 0 & d & 0 & e \\ & & b & 0 & f & 0 \\ & & & b & 0 & f \\ & & & & c & 0 \\ & & & & & c\end{array}\right)$

$60 \quad\left(\begin{array}{cccccc}a & b & c & d & d & c \\ & a & b & c & d & d \\ & & a & b & c & d \\ & & & a & b & c \\ & & & & a & b \\ & & & & & a\end{array}\right)$ with $a+2 b+2 c+2 d=0$

61

$\left(\begin{array}{cccccc}a & b & c & -\frac{1}{2} a & -b-c & -b-c \\ & a & b & c & -\frac{1}{2} a & -b-c \\ & & a & b & c & -\frac{1}{2} a \\ & & & a & b & c \\ & & & & a & b \\ & & & & & a\end{array}\right)$

$\left(\begin{array}{cccccc}a & b & b & b & b & b \\ & a & b & -b & -b & b \\ & & a & b & -b & -b \\ & & & a & b & -b \\ & & & & a & b \\ & & & & & a\end{array}\right)$

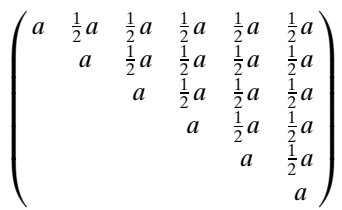

89

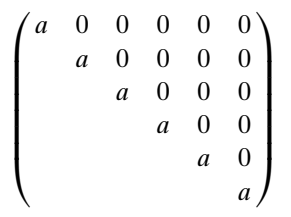

91
No.

10

23

62

88

90
Standard tensor

$$
\begin{gathered}
\left(\begin{array}{cccccc}
a & -\frac{1}{2} a & d & -d-g & e & h \\
& a & g & d & -e-h & e \\
& & b & -\frac{1}{2} b & f & i \\
& & & b & -f-i & f \\
& & & & c & -\frac{1}{2} c \\
& & & & & c
\end{array}\right) \\
\left(\begin{array}{cccccc}
a & -\frac{1}{2} a & d & -\frac{1}{2} d & e & -\frac{1}{2} e \\
& a & -\frac{1}{2} d & d & -\frac{1}{2} e & e \\
& & b & -\frac{1}{2} b & f & -\frac{1}{2} f \\
& & & b & -\frac{1}{2} f & f \\
& & & & c & -\frac{1}{2} c \\
& & & & & c
\end{array}\right)
\end{gathered}
$$

The other metrical tensors are either the most general with 21 independent components or can be written as the direct sum of independent tensors of lower dimensions.

when $l=n$ (only eigenvalues +1 ) and

$$
\overline{1_{m}}
$$

when $m=n$ (only eigenvalues -1 ). The order is such that

$$
q_{i} \geq q_{i+1} \geq \ldots, \quad p_{i} \leq p_{i+1} \quad \text { if } q_{i}=q_{i+1} .
$$

(II) For consistency with current practice, $m$ should be used for $\overline{1_{1}}, 2$ is used exceptionally instead of $\overline{1_{2}}$, and $\overline{1}$ is used instead of $\overline{1_{3}}$.
(III) In the case of a crystallographic orthogonal transformation, a short form is obtained by combining conjugate blocks with the same $q$ into the symbol $[q]$. This represents the sequence

$$
[q]=q^{p_{1}} \ldots q^{p_{e}},
$$

where $p_{1}, \ldots, p_{e}$ are the $\Phi(q) / 2$ integers that are coprime with $q$ and smaller than $q / 2$.

(IV) The symbol does not depend on the number of eigenvalues +1 . However, if there are only eigenvalues 
+1 and no others, then the transformation should be denoted by $1_{n}$. If it is desirable to indicate explicitly the dimension of the transformation as many digits 1 may be added as there are eigenvalues equal to +1 .

(V) Symbols for geometric crystal classes are symbols for representative point groups. The following symbols should be used in point groups that are the subdirect product of point groups in at most three dimensions.

(i) A point group in $n$ dimensions that can be written as the direct product of point groups acting in mutually perpendicular subspaces is called an external product of $K_{1}, K_{2}, \ldots$ and is denoted by $K_{1} \perp K_{2} \perp \ldots$ A subdirect product of $K_{1}, K_{2}, \ldots$ is a subgroup of the external product $K_{1} \perp K_{2} \perp \ldots$.

(ii) If the point group is reducible into block form with blocks of dimension three or less and not an external product of point groups, the following symbols are chosen.

For convenience, we consider the case where the point group $K$ is a subgroup of $K_{1} \perp K_{2}$. Generalization to the case with more components is straightforward. The symbol consists of a pair $H_{1}\left(H_{2}\right)$, where $H_{i}$ is the symbol for a two- or three-dimensional point group $K_{i}$ or such a symbol with digits 1 added so that $(a)$ the number of alphanumeric characters is the same in both symbols, $(b)$ the $m$ th symbol in $H_{1}$ is associated with the $m$ th symbol in $H_{2}$, and $(c)$ the elements of $H_{i}$ that are paired with a digit 1 in the other symbol generate an invariant subgroup.

(iii) Simplified symbols are chosen for the groups $K$ that are the direct product of a subgroup $H$ of index two and the central inversion $\overline{1_{n}}$. Such a group is denoted by $H \times \overline{1_{n}}$.

(VI) Recommended symbols for families are the same as those for corresponding holohedral point groups.

(VII) The family order is first according to the number of free parameters in the metric tensor (in decreasing order), and second according to the order of the holohedral point group (in increasing order).

(VIII) The standard basis for each family is chosen such that the reducibility becomes maximally evident. Orthogonal invariant subspaces are ordered according to decreasing orders of the corresponding point groups. The conventional cell for a lattice system is the cell of the corresponding family. As an alternative, a special basis for each lattice system in the family may be used. The recommended bases and metric tensors for four-, five- and six-dimensional families are given in Tables 4,7 and 9.

We thank Subcommittee advisors G. Chapuis, N. D. Mermin, R. Veysseyre and E. J. W. Whittaker for useful comments. We thank, in particular, former Subcommittee member H. Wondratschek for many important contributions.

\section{References}

Ascher, E. \& Janner, A. (1965). Helv. Phys. Acta, 38, 551-572. Ascher, E. \& Janner, A. (1968). Commun. Math. Phys. 11, 138-167.

Bieberbach, L. (1911) Math. Ann. 70, 297-336, 400-412.

Brown, H., Bülow, R., Neubüser J., Wondratschek, H. \& Zassenhaus, H. (1978). Crystallographic Groups of FourDimensional Space. New York: Wiley.

Fedorov, E. S. (1891a). Proc. St Petersburg Mineral. Soc. 28, 1-146. (In Russian.)

Fedorov, E. S. (1891b). Z. Kristallogr. Mineral. 20, 25-75.

Heesch, H. (1929). Z. Kristallogr. 71, 95-102.

Hermann, C. (1949). Acta Cryst. 2, 139-145.

Hurley, A. C. (1951). Proc. Cambridge Philos. Soc. 47, 650-661.

Hurley, A. C. (1966). Quantum Theory of Atoms, Molecules and the Solid State, edited by P. O Löwdin, pp. 571-586. New York: Academic Press.

International Tables for Crystallography (1995). Vol. A, SpaceGroup Symmetry, 4th revised ed., edited by Th. Hahn. Dordrecht: Kluwer Academic Publishers.

Janner, A., Janssen, T. \& de Wolff, P. M. (1983). Acta Cryst. A39, 658-666.

Janssen, T. (1969). Physica (Utrecht), 42, 71-92.

Opgenorth, J., Plesken, W. \& Schulz, T. (1998). Acta Cryst. A54, 517-531.

Plesken, W. \& Hanrath, W. (1984). Math. Comput. 43, 573-587.

Plesken, W. \& Pohst, M. (1977). Math. Comput. 31, 536-551, 551-573.

Schoenflies, A. (1891). Krystallsysteme und Krystallstructur. Leipzig: Teubner. Reprinted (1984) Springer, Berlin.

Veysseyre, R. (1998). Private communication.

Weigel, D., Phan, T. \& Veysseyre, R. (1987). Acta Cryst. A43, 294-304.

Weigel, D., Phan, T. \& Veysseyre, R. (1993). Acta Cryst. A49, 486-492.

Wondratschek, H. (1998). Private communication.

Yamamoto, A. (1996). Acta Cryst. A52, 509-560. 